\title{
AXL/MERTK inhibitor ONO-7475 potently synergizes with venetoclax and overcomes venetoclax resistance to kill FLT3-ITD acute myeloid leukemia
}

\author{
Sean M. Post, ${ }^{1}$ Huaxian Ma,, Prerna Malaney, ${ }^{1}$ Xiaorui Zhang, ${ }^{1}$ Marisa J.L. Aitken, ${ }^{1}$ Po Yee \\ Mak, ${ }^{1,2}$ Vivian R. Ruvolo, ${ }^{1,2}$ Tomoko Yasuhiro, ${ }^{3}$ Ryohei Kozaki, ${ }^{3}$ Lauren E. Chan, ${ }^{1}$ Lauren B. \\ Ostermann, ${ }^{1,2}$ Marina Konopleva, ${ }^{1}$ Bing Z. Carter, ${ }^{1,2}$ Courtney DiNardo, ${ }^{1}$ Michael D. Andreeff,, \\ Joseph D. Khoury, ${ }^{4 \#}$ and Peter P. Ruvolo ${ }^{1,2 \#}$ \\ 'Department of Leukemia, ${ }^{2}$ Section of Molecular Hematology, The University of Texas MD \\ Anderson Cancer Center, Houston, TX, USA; ${ }^{3}$ Ono Pharmaceutical Co. Ltd., Research Center \\ of Oncology, Osaka, Japan and ${ }^{4}$ Department of Hematopathology, The University of Texas \\ MD Anderson Cancer Center, Houston, TX, USA
}

\#JDK and PPR contributed equally as co-senior authors.

\author{
Correspondence: \\ Joseph D. Khoury \\ JKhoury@mdanderson.org \\ Sean M. Post \\ SPost@mdanderson.org \\ Received: January 13,202 \\ Accepted: October 28, 2021 \\ Prepublished: November 4, 2021. \\ https://doi.org/10.3324 haematol.2021.278369 \\ @2022 Ferrata Storti Foundation \\ Haematologica material is published under a CC

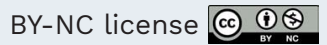

\begin{abstract}
FMS-like Tyrosine Kinase 3 (FLT3) mutation is associated with poor survival in acute myeloid leukemia (AML). The specific Anexelekto/MER Tyrosine Kinase (AXL) inhibitor, ONO-7475, kills FLT3-mutant AML cells with targets including Extracellular-signal Regulated Kinase (ERK) and Myeloid Cell Leukemia 1 (MCL1). ERK and MCL1 are known resistance factors for Venetoclax (ABT-199), a popular drug for AML therapy, prompting the investigation of the efficacy of ONO-7475 in combination with ABT-199 in vitro and in vivo. ONO-7475 synergizes with ABT-199 to potently kill FLT3-mutant acute myeloid leukemia cell lines and primary cells. ONO-7475 is effective against ABT-199-resistant cells including cells that overexpress MCL1. Proteomic analyses revealed that ABT-199-resistant cells expressed elevated levels of pro-growth and anti-apoptotic proteins compared to parental cells, and that ONO-7475 reduced the expression of these proteins in both the parental and ABT-199-resistant cells. ONO-7475 treatment significantly extended survival as a single in vivo agent using acute myeloid leukemia cell lines and PDX models. Compared to ONO-7474 monotherapy, the combination of ONO-7475/ABT-199 was even more potent in reducing leukemic burden and prolonging the survival of mice in both model systems. These results suggest that the ONO-7475/ABT-199 combination may be effective for AML therapy.
\end{abstract}

\section{Introduction}

Internal tandem duplication (ITD) mutation in FMS-like Tyrosine Kinase 3 (FLT3) occurs in $\sim 25 \%$ of newly diagnosed acute myeloid leukemia (AML) patients and is associated with poor survival.1-4 Therapeutic strategies for FLT3-ITD AML patients include the use of broad tyrosine kinase inhibitors (TKIs) such as Midostaurin and Gilteritinib, whose selectivity includes FLT3. ${ }^{1}$ TKIs have limited efficacy and resistance mechanisms involving FLT3 alterations, such as D835, mutations make their use challenging. ${ }^{1-4}$ The B-cell lymphoma 2 (BCL2) inhibitor ABT-199 (venetoclax) is being evaluated in numerous clinical trials for AML and is typically combined with other agents as ABT-199 has limited effectiveness as a single agent. ${ }^{5,6}$ Similar to other BH3-mimetic drugs, ABT-199 induces cross-activation of extracellular-signal regulated kinase $1 / 2 \quad$ (ERK1/2) and downstream induction of myeloid cell leukemia-1 (MCL1); a survival factor for AML cells with FLT3-ITD mutations. $^{7-13}$ Thus, agents that ablate FLT3 and ERK1/2 signaling and block BCL2 are critical therapies for FLT3ITD AML. A recent study using FLT3-ITD AML models demonstrated that Midostaurin or Gilteritinib synergize with ABT-199. ${ }^{14}$ However, Gilteritinib induces ERK activation and promotes RAS mutations while Midostaurin promotes the

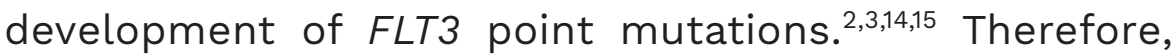
novel treatment options that do not activate the RAS/ERK pathway or select for FLT3 alterations are needed.

Various studies support a role for the tyrosine kinase receptor, Anexelekto (AXL), as a critical component in FLT3ITD signaling. ${ }^{16-19}$ ONO-7475 is a specific inhibitor of AXL and MER Tyrosine Kinase (MERTK), and our group has shown it potently kills FLT3-ITD AML cells. ${ }^{19}$ Notably, ONO7475 suppresses ERK1/2 phosphorylation and reduces 
MCL-1 expression. Therefore, we hypothesized that ONO7475 can simultaneously target BCL2 and the FLT3 signaling pathways while mitigating the deleterious alterations reported with other TKIs. ${ }^{2,3,13,14}$ In this report, we examine the efficacy of the ONO-7475/ABT-199 combination using in vitro and in vivo models of $A M L$ and test the ability of ONO-7475 to overcome ABT-199 resistance or MCL-1 overexpression (either overexpression of wild-type (WT) or gain-of-function (GOF) mutant MCL-1) in FLT3-ITD cells.

\section{Methods}

\section{Cell lines}

MOLM13 and OCI-AML3 cells were purchased from DSMZ (Braunschweig, Germany). MV4;11 and THP-1 cells were purchased from ATCC (Manassas, VA, USA). MOLM13 luc/gfp cells were generated as previously described. ${ }^{19}$ MV4;11 cells rendered resistant to ABT-199 (MV4;11 VenR) were developed by long-term exposure to ABT-199, as previously described. ${ }^{10}$ MV4;11 stable cell lines overexpressing MCL-1 (wild-type and GOF mutant S159A) and MOLM13 cell lines overexpressing BCL2 (wildtype and phospho-mutants S70A and S70E) with corresponding control plasmids were created, as previously described. ${ }^{7}$ Cells were cultured at $21 \% \mathrm{O}_{2}$ (normoxia) or $1 \% \mathrm{O}_{2}$ (hypoxia) and $5 \% \mathrm{CO}_{2}$ at $37^{\circ} \mathrm{C}$.

\section{Reagents}

ONO-7475 was supplied by Ono Pharmaceutical Co. Ltd. (Osaka, Japan); ABT-199 was purchased from LC Laboratories (Woburn, MA, USA); UMI-77 was purchased from Selleck Chem (Houston, TX, USA); stock solutions for in vitro studies were prepared with DMSO (Sigma-Aldrich, St. Louis, MO, USA). For animal studies, ONO-7475 was prepared in $0.1 \%$ Tween 80 and ABT-199 was prepared in 10\% ethanol/30\% phosphal 50/60\% PEG 400.

\section{Cell viability}

Cells were incubated with vehicle or varying doses of ONO-7475 and/or ABT-199, then stained with Annexin V APC (BD Biosciences, San Jose, CA, USA) and DAPI (BD Biosciences, San Jose, CA, USA). Flow cytometry was performed using the Galios561 (Beckman Coulter, Brea, CA, USA). Further, cell viability was analyzed using the Vi-Cell Cell Viability Analyzer (Beckman Coulter).

\section{Immunoblot analysis}

Cells were incubated with vehicle ONO-7475 and/or ABT199 and then lysed. Total protein was fractionated by SDS/PAGE. Immunoblot analysis was performed with antibodies listed in the Online Supplementary Methods and imaged as previously described. ${ }^{20}$ Tubulin and $\beta$-actin were used as loading controls.

\section{qRT-PCR}

RNA was isolated and qRT-PCR performed as described in Online Supplementary Methods.

\section{Patient samples}

Primary FLT3-ITD AML samples were acquired in accordance with regulations and protocols approved by the Institutional Review Board of the MD Anderson Cancer Center (MDACC). Informed consent was obtained in accordance with the Declaration of Helsinki. Patient characteristics are provided in Online Supplementary Table S2.

\section{Human AML xenograft in vivo model}

Human xenograft experiments were approved by the Institutional Animal Care and Use Committee at MDACC. The efficacy of the ONO-7475/ABT-199 combination in an in vivo FLT3-ITD AML xenograft model was tested using MOLM13 cells expressing luciferase/GFP and the AML PDX model 3028566 in NSG mice. The AML PDX also harbored NPM1 and DNMT3A mutations. For both models, drugs were given five days a week by oral gavage (ONO-7475 at $10 \mathrm{mg} / \mathrm{kg}$ and ABT-199 at $100 \mathrm{mg} / \mathrm{kg}$ ). Leukemia burden was assessed by IVIS imaging for MOLM13 and flow cytometry to measure human $\mathrm{CD} 45^{+}$cells for the PDX model.

\section{Statistical analyses}

All in vitro experiments were conducted in triplicate. The combination index $(\mathrm{Cl})$, based on the Chou-Talalay method $^{21}$ and determined by CalcuSyn software (BIOSOFT), was expressed as $\mathrm{Cl}$ values obtained at the effective doses (ED) of $50 \%, 75 \%$, and $90 \%$ in the population exposed to the different agents. $\mathrm{Cl}<1$ was considered synergistic, $\mathrm{Cl}=1$ additive, and $\mathrm{Cl}>1$ antagonistic. Statistical differences between groups were determined using either a Student's $t$-test or one-way ANOVA with Dunnett's posttest. $P \leq 0.05$ was considered statistically significant.

\section{Results}

\section{Combination of ONO-7475 with ABT-199 is effective in vitro against FLT3-ITD AML cells}

MV4;11 cells were treated with vehicle or varying doses of ONO-7475 +/- ABT-199. After 72 hours, cell viability and cell number were determined by flow cytometry using Annexin $V$ antibody and DAPI. Both ONO-7475 (10 nM) and ABT-199 (30 nM) showed similar efficacy as single agents with a $\sim 60 \%$ reduction in cells (Figure $1 \mathrm{~A}$ ). While $10 \mathrm{nM}$ ONO-7475 did not induce apoptosis, 30 nM ABT-199 resulted in $\sim 35 \%$ apoptosis (Online Supplementary Figure $1 A$ ). Interestingly, the combination of $10 \mathrm{nM}$ ONO-7475 with 30 nM ABT-199 nearly eliminated MV4;11 cells ( 98\% reduction of viable cells; Figure $1 \mathrm{~A}$ ) with $>84 \%$ of cells undergoing apoptosis (Online Supplementary Figure $1 \mathrm{~A}$ ). 
The combination of $50 \mathrm{nM}$ ONO-7475 with $30 \mathrm{nM}$ ABT- 199 reduced the viability of $M V 4 ; 11$ cells $>99 \%$ (Figure $1 A$ ) and induced $\sim 98 \%$ apoptosis (Online Supplementary Figure $1 A$ ). MOLM13 cells showed a $60 \%$ reduction in viable cells with 10 nM ONO-7475 with limited induction (<15\%) of apoptosis (Figure 1B and Online Supplementary Figure 1B). Likewise, the combination of $50 \mathrm{nM}$ ONO-7475 with $30 \mathrm{nM}$ ABT-199 in MOLM13 cells resulted in $>99 \%$ reduction in vi- ability (Figure 1B) with $~ 77 \%$ of cells undergoing apoptosis (Online Supplementary Figure 1B). For FLT3 WT THP-1 and OCI-AML3 cells, ONO-7475 did not significantly augment cell reduction or apoptotic induction by ABT-199 (Online Supplementary Figures S2A-2D).

To determine whether ONO-7475 synergizes with ABT-199, MOLM13 and MV4;11 cells were treated with vehicle or varying doses of ONO-7475 and/or ABT-199 for 72 hours.
A

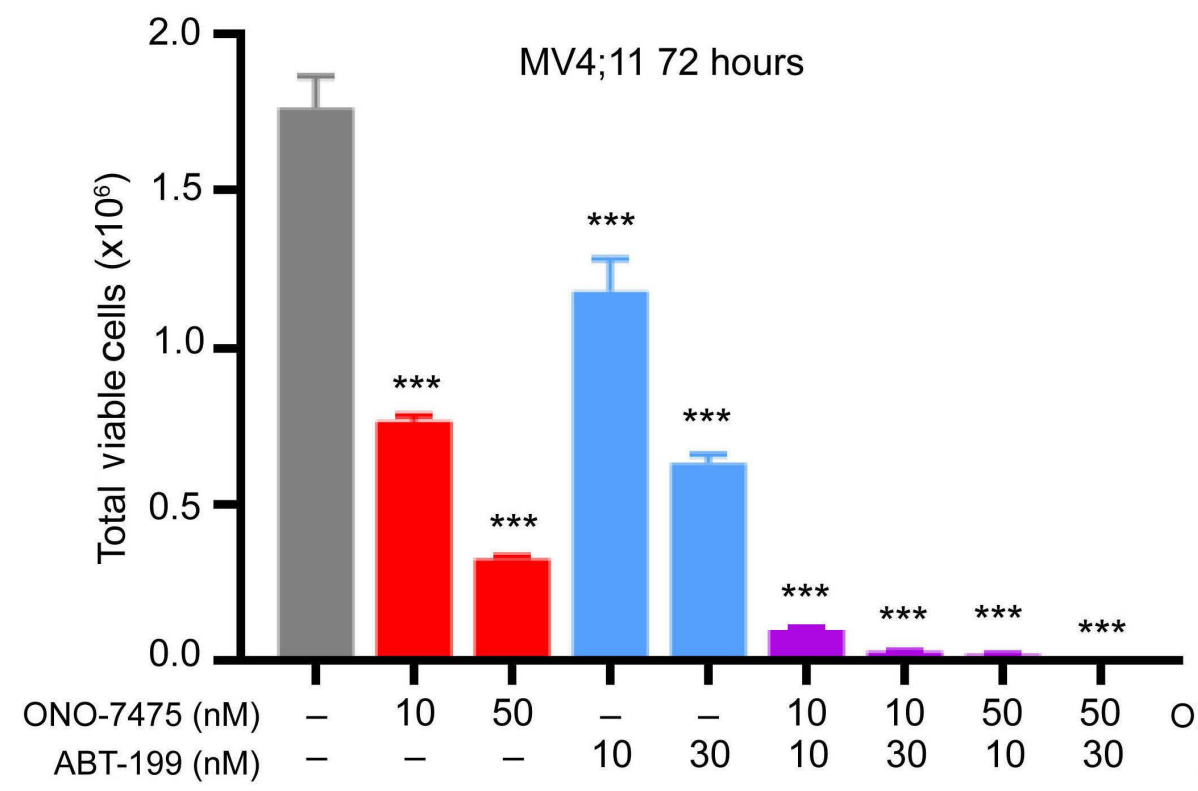

C

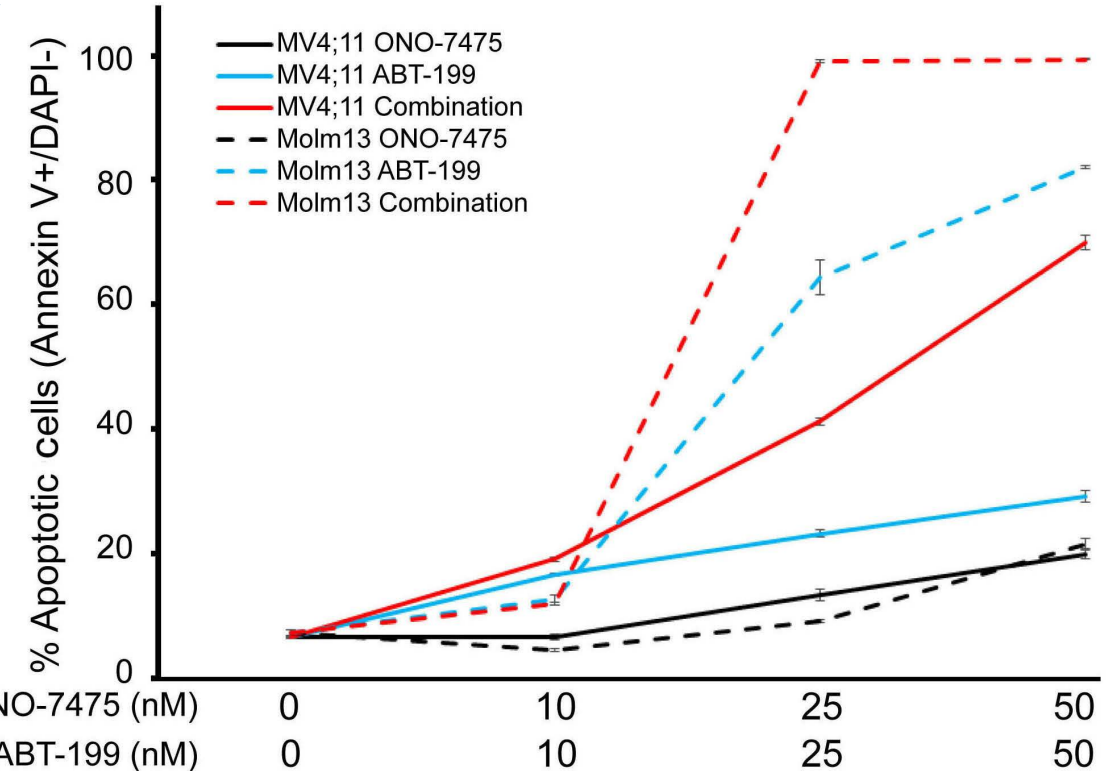

MV4;11 CI Values at:

ED50 ED75 ED90

$0.18937 \quad 0.08279 \quad 0.04449$
MOLM13 CI Values at:

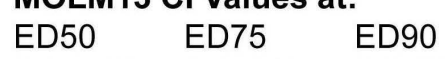

$\begin{array}{lll}0.15125 & \text { ED75 } & \text { ED90 } \\ 0.08818 & 0.05335\end{array}$
B

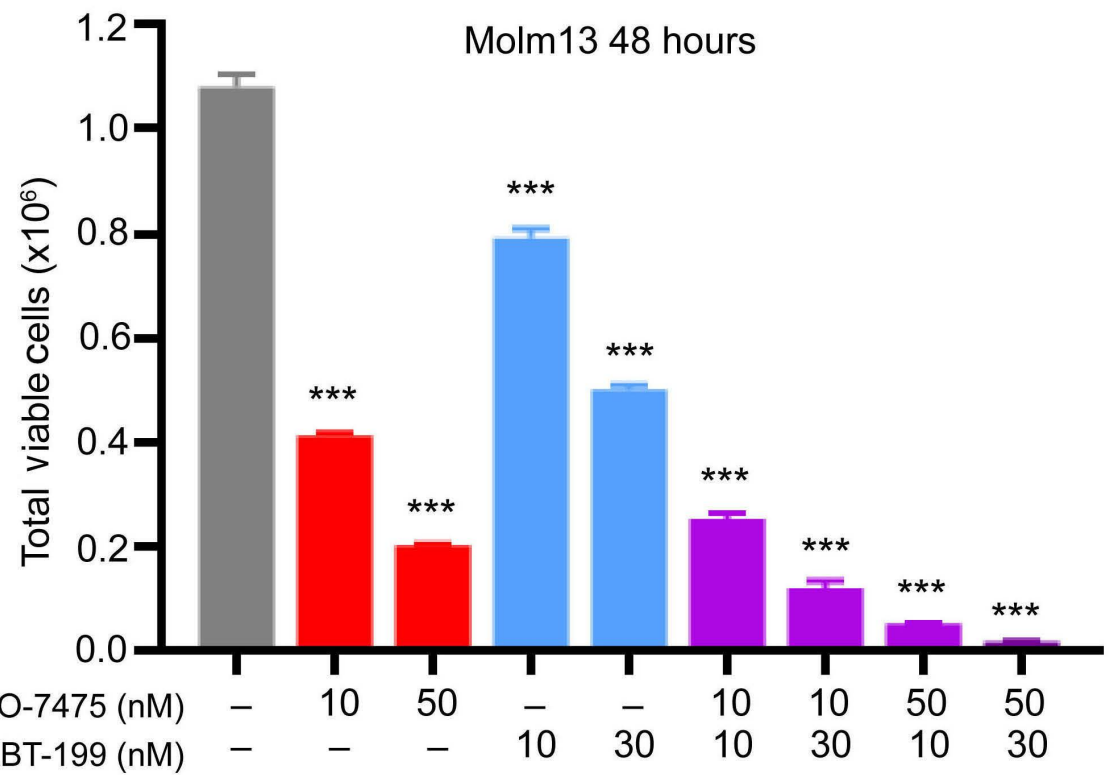

D

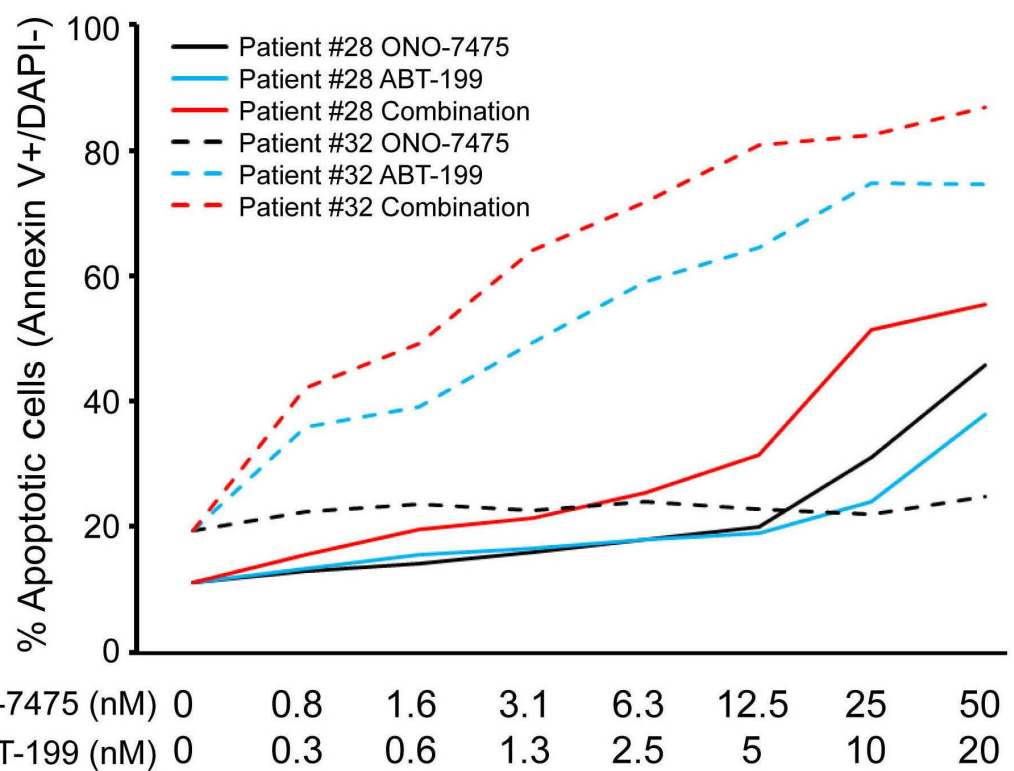

Patient \#28 CI Values at:

ED50 ED75 ED90

Patient \#32 Cl Values at:

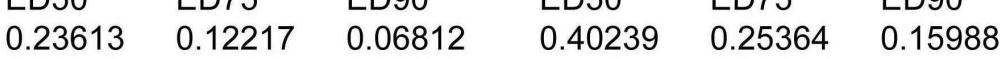

Figure 1. ONO-7475 synergizes with ABT-199 to potently kill FLT3-ITD AML cell lines and primary cells. MV4;11 and MOLM13 cells were treated with vehicle (0.2\% DMSO), $10 \mathrm{nM}$ or $50 \mathrm{nM}$ ONO-7475, $10 \mathrm{nM}$ or $30 \mathrm{nM}$ ABT-199, or combinations of the two agents for 72 hours (MV4;11) or 48 hours (MOLM13). Viable total cells (A) for MV4;11; (B) for MOLM13 were determined by flow cytometry using counting beads, Annexin V, and DAPI. One-way ANOVA with Dunnett's post-test was performed to determine significance $\left({ }^{*} P<0.033 ; * \star P<0.002 ; * \star \star P<0.001\right)$. (C) MV4;11 cells and MOLM13 were treated with vehicle (0.2\% DMSO), $10 \mathrm{nM}, 25 \mathrm{nM}$, or $50 \mathrm{nM}$, ONO-7475 or $10 \mathrm{nM}, 25 \mathrm{nM}$, or $50 \mathrm{nM}$ ABT-199, or combinations of the two agents in a 1:1 ratio for 72 hours. Apoptosis was determined by flow cytometry using Annexin $\mathrm{V}$ and DAPI stains. $\mathrm{Cl}$ index was determined using CalcuSyn software for combinations at effective dose (ED) 50, ED75, and ED90. $\mathrm{Cl}<1$ was considered synergistic, $\mathrm{Cl}=1$ additive, and $\mathrm{Cl}>1$ antagonistic. (D) Peripheral blood cells from AML patients \#28 and \#32 were treated with vehicle (0.2\% DMSO), various doses of ONO-7475 or various doses of ABT-199, or combinations of the two agents in a ratio of $1 \mathrm{nM}$ ABT-199: $2.5 \mathrm{nM}$ ONO-7475 ratio for 42 hours. Apoptosis was determined by flow cytometry using Annexin $\mathrm{V}$ and DAPI stains. Cells were gated for CD45 cells using CD45 antibody. CI index was determined using CalcuSyn software for combinations at effective dose (ED) 50, ED75, and ED90. Cl < 1 was considered synergistic, $\mathrm{Cl}=1$ additive, and $\mathrm{Cl}>1$ antagonistic. 
Synergy was determined by measuring $\mathrm{Cl}$, based on the Chou-Talalay method..$^{21} \mathrm{Cl}$ values were obtained at the ED $50 \%, 75 \%$, and $90 \%$ in the population exposed to the different agents. We observed that ONO-7475 synergizes with ABT-199, resulting in a $\mathrm{Cl}$ index below 1.0 at ED50, ED75, and ED90 (Figure 1C). In fact, $\mathrm{Cl}$ was $<0.1$ in both cell lines at ED75 and ED90.

To determine the efficacy of ONO-7475/ABT-199 combination in primary FLT3-ITD AML cells, fresh cells from the peripheral blood of two primary AML samples were tested. Cells were treated for 48 hours with varying doses of ONO$7475+/-A B T-199$. As a single agent, ONO-7475 was slightly more effective at inducing apoptosis compared to ABT-199 in the sample from Patient \#28 (Figure 1D). Importantly, ONO-7475 and ABT-199 acted synergistically to enhance apoptosis $(\mathrm{Cl}=0.12$, ED75 and 0.07, ED90, Figure 1D). Interestingly, ONO-7475 was ineffective as a single agent in cells derived from Patient \#32 while ABT-199 alone promoted apoptosis (Figure 1D). However, when combined, ONO7475/ABT-199 synergized to activate apoptosis $\mathrm{Cl}=0.25$, ED75 and 0.16, ED90, Figure 1D). Given that AXL expression is, in part, regulated by hypoxia, ${ }^{22}$ we next evaluated the efficacy of these agents in $1 \% \mathrm{O}_{2}$. Here, we observed that these combinations remained effective and synergistic in hypoxic conditions (Online Supplementary Figure S3A-B). We next interrogated alterations in MCL-1 expression and ERK phosphorylation following treatment with vehicle, single agent, or combinations of each agent (Online Supplementary Figure $S 4 A$ ). We observed that low-dose ONO7475 had minimal effects on MCL-1 expression in either cell line, although the ONO-7475/ABT- 199 combination did reduce MCL-1 in MV4;11 cells (Online Supplementary Figure S4A). While $10 \mathrm{nM}$ ONO-7475 in combination with $30 \mathrm{nM}$ ABT-199 effectively killed MOLM13 cells (Figure 1B and Online Supplementary Figure 1B), this combination did not affect MCL-1 expression (Online Supplementary Figure 4A), suggesting that suppression of MCL-1 is not required for ONO-7475/ABT-199-mediated killing. Interestingly, ERK phosphorylation was suppressed by $10 \mathrm{nM}$ ONO-7475 alone and in combination with ABT-199 in both cell lines (Online Supplementary Figure $4 A$ ), suggesting ONO-7475 may synergize with ABT-199 to impede cell proliferation.

\section{ONO-7475 is equally effective against parental and ABT-199-resistant MV4;11 cell growth}

We previously developed MV4;11 cells that are $>100$ fold more resistant to ABT-199 than parental cells. ${ }^{10}$ MV4;11 parental and ABT-199-resistant cells were treated with vehicle or varying amounts of ONO-7475 for 72 hours. Both $10 \mathrm{nM}$ and $50 \mathrm{nM}$ ONO-7475 were equally efficient in reducing parental and ABT-199-resistant cell growth (Figure $2 \mathrm{~A}$ ) and inducing apoptosis (Figure 2B).

To determine whether ONO-7475 synergizes with ABT-199 in MV4;11 ABT-199-resistant cells, cells were treated with vehicle, ONO-7475, and/or ABT-199 for 72 hours. Apoptosis was determined by flow cytometry. The highest tested dose of ABT-199 (5000 nM) had little effect on apoptosis in resistant cells while the highest tested dose of ONO$7475(50 \mathrm{nM})$ resulted in only modest apoptosis (Figure 2C). However, the combination of ONO-7475 and ABT-199 was able to induce greater apoptosis with lower doses: $50 \%$ apoptotic induction with $25 \mathrm{nM}$ ONO- 7475/2500 nM ABT-199 and $~ 90 \%$ apoptosis with $50 \mathrm{nM}$ ONO-7475/5000 nM ABT-199, (CI ED50, ED75, and ED90 below 0.25, Figure 2C). Increased MCL-1 expression is a known resistance mechanism for ABT-199 and interestingly, the treatment of ABT-199-resistant cells with UMI-77, an MCL-1 inhibitor, (and/or ONO-7475) also significantly reduced cell viability (Online Supplementary Figure S4B).

\section{Proteomic analyses reveal similar ONO-7475-dependent suppression of pro-growth and pro-survival targets in parental and ABT-199-resistant cells}

To determine ONO-7475's effect on growth and survival signaling pathways in parental and ABT-199-resistant cells, we initially utilized RPPA to profile proteomes of MV4;11 parental and ABT-199-resistant cells following treatment. These analyses revealed alterations in protein expression between untreated MV4;11 parental and ABT199-resistant cells (Online Supplementary Figure S5A).

Critically, untreated MV4;11 ABT-199-resistant cells had a significant increase in the expression of pro-growth and survival signaling compared to parental cells. In fact, BRAF levels, an upstream positive regulator of ERK pathways ${ }^{23}$ were markedly higher in the resistant cells, suggesting this increase may stimulate survival pathways in these cells (Figure 3A). Further, we observed elevated expression of the pro-growth translational proteins, $\mathrm{S} 6$ and $\mathrm{p}-\mathrm{S} 6$ (Figure $3 \mathrm{C}$ ).

We once again used RPPA to evaluate ONO-7475-mediated expression changes between resistant and parental cell lines. Following treatment with $100 \mathrm{nM}$ ONO-7475 for 24 hours, most proteins were similarly altered in both the resistant and parental cell lines (Online Supplementary Figure S5B). Critically, we observed that ONO-7475 treatment significantly reduced the expression of B-RAF, p-S6, pERK, and MCL1 (Figure 3A-D). Our observations that $\mathrm{p}-\mathrm{S6}$, p-ERK, and MCL1 were suppressed in both resistant and parental lines following ONO-7475 treatment, suggest that ABT-199 resistances may be overcome by ONO-7475/AXL inhibition.

\section{Overexpression of BCL2 but not MCL-1 protects cells from ONO-7475}

MCL-1 overexpression protects cells from ABT-199 and other $\mathrm{BH} 3$ mimetic agents. ${ }^{7,8,10}$ To evaluate the relationship between MCL-1 levels and sensitivity to ONO-7475, we used MV4;11 cells containing either an EV control plasmid, WT 
A

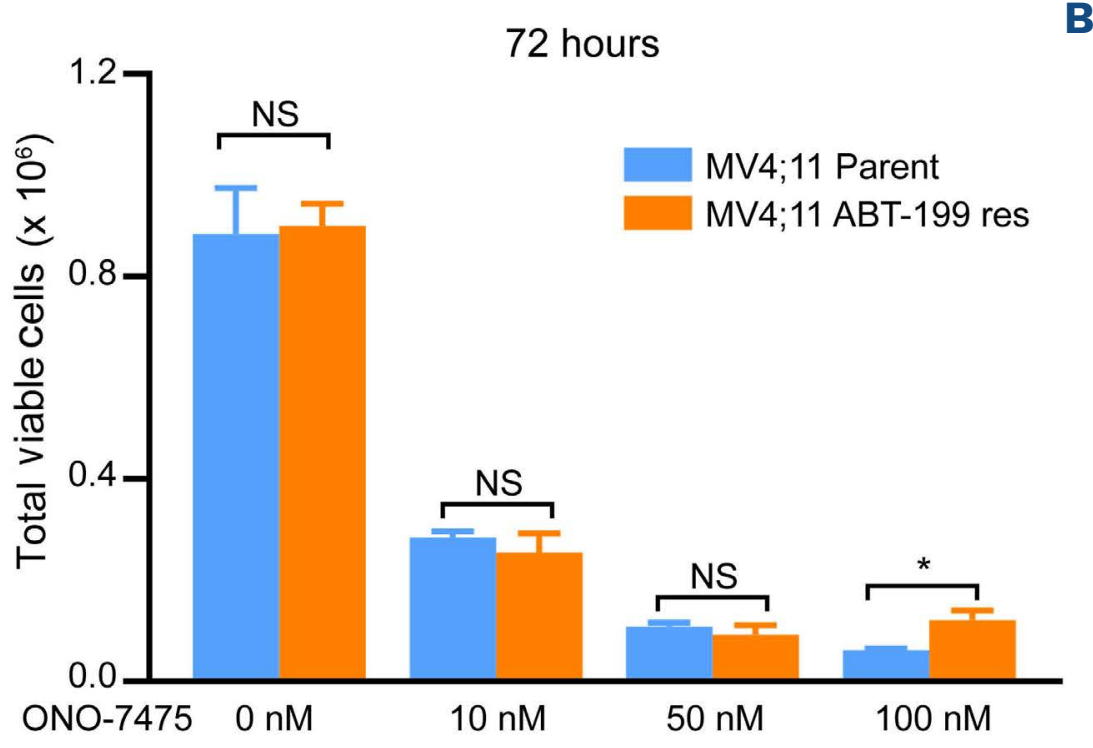

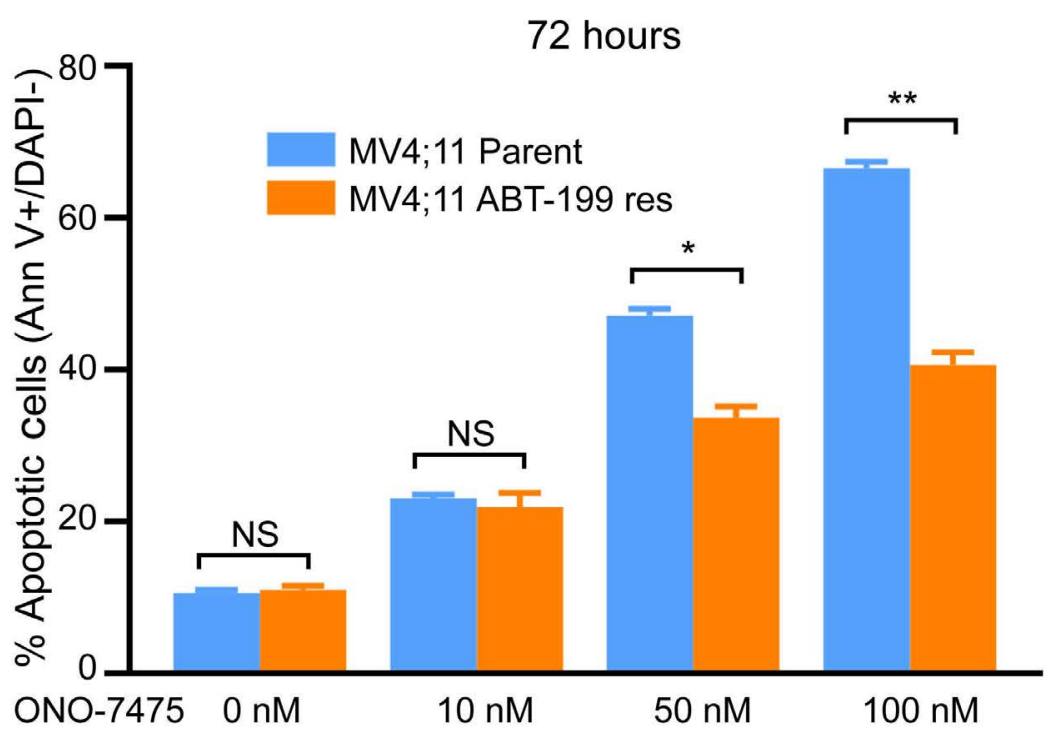

C

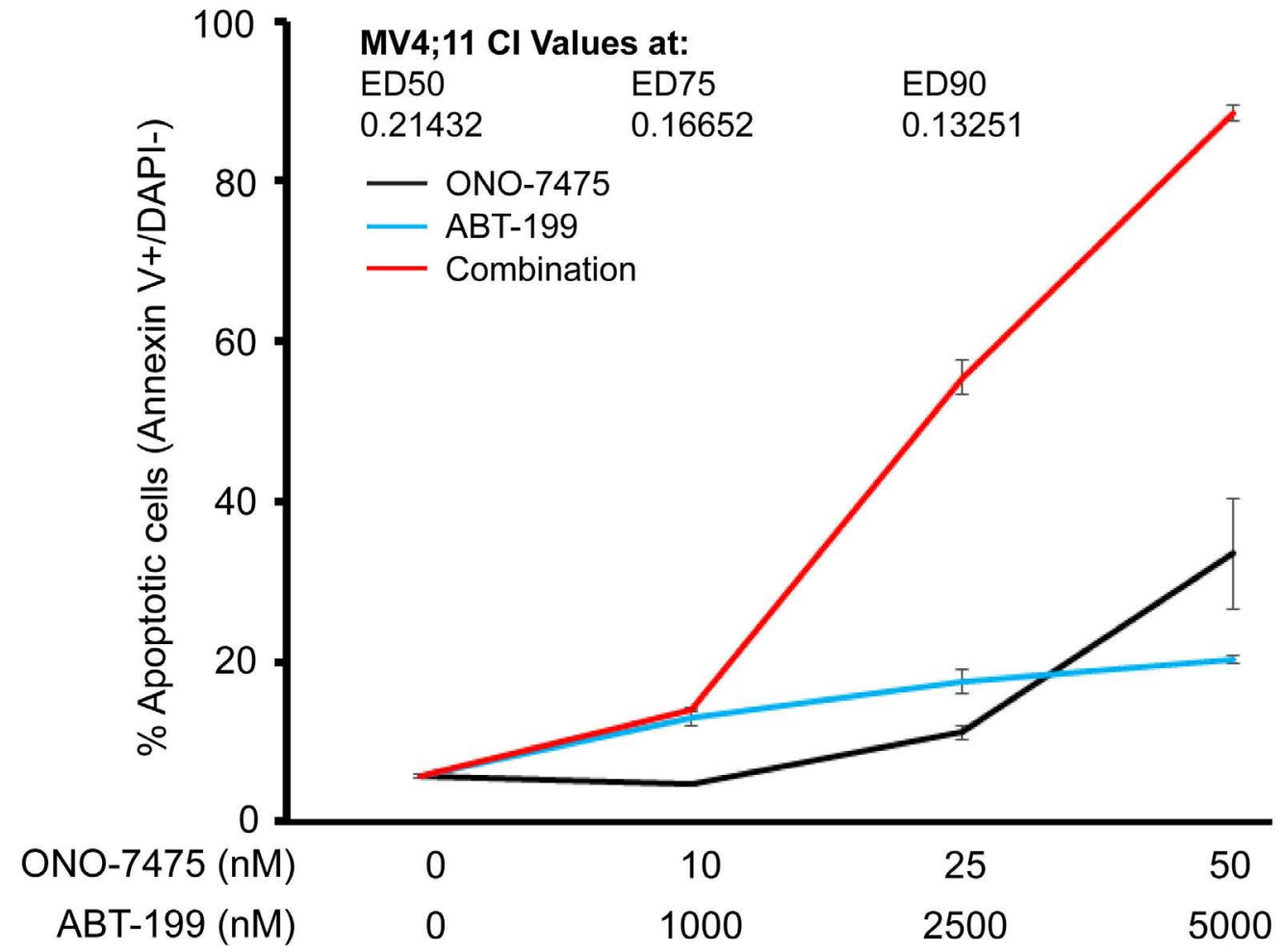

Figure 2. ONO-7475 impacts growth of ABT199-resistant MV4;11 cells and parental cells. MV4;11 parental and MV4;11 ABT-199-resistant cells were treated with vehicle (0.1\% DMSO), $10 \mathrm{nM}, 50 \mathrm{nM}$, or $100 \mathrm{nM}$ ONO-7475 for 72 hours. Viable total cells $(A)$ and percentage of apoptotic cells (B) were determined by flow cytometry using counting beads, Annexin V, and DAPI. Student's t-test was performed to determine significance $(* P<0.05 ; * * P<0.001$; $\star * * P<0.0001)$. (C) MV4;11 ABT-199-resistant cells were treated with vehicle (0.2\% DMSO), $10 \mathrm{nM}, 25 \mathrm{nM}$, or $50 \mathrm{nM}$ ONO-7475 or $1000 \mathrm{nM}$, $2500 \mathrm{nM}$, or $5000 \mathrm{nM}$ ABT-199, or combinations of the two agents in ratio of $1 \mathrm{nM}$ ONO7475: 100 nM ABT-199 for 72 hours. Apoptosis was determined by flow cytometry using Annexin $\mathrm{V}$ and DAPI stains. $\mathrm{Cl}$ index was determined using Calcusyn software for combinations at effective dose (ED) 50, ED75, and ED90. $\mathrm{Cl}<1$ was considered synergistic, $\mathrm{Cl}=1$ additive, and $\mathrm{Cl}>1$ antagonistic.
MCL-1 or GOF MCL-1 mutant (S159A), which promotes MCL1 protein stability (Figure $4 \mathrm{~A}$ ). ${ }^{24,25}$ These cells were treated with varying doses of ABT-199 or ONO-7475 for 72 hours: EV-control cells were sensitive to $30 \mathrm{nM}$ ABT-199 whereas both WT and GOF mutant MCL-1 OE cells were resistant to ABT-199 (Figures 4B and 4C). While, on average, $87 \%$ of EVcontrol cells were killed by ABT-199, only $32 \%$ of WT MCL1 OE cells and $19 \%$ of GOF mutant MCL-1 OE cells were. On the other hand, EV-control, WT MCL-1 OE, and GOF mutant MCL-1 OE cells were all similarly sensitive to ONO-7475 (Figure 4B). On average, induction of apoptosis in EV cells, WT MCL-1 cells and GOF mutant MCL-1 OE cells by $50 \mathrm{nM}$ ONO-7475 is $~ 85 \%$ in all variants. The failure of MCL-1, including the GOF mutant, to protect these cells from ONO7475 or alter cellular AXL levels suggests that the ONO-7475 mechanism of action is independent of MCL-1 expression levels (Figure 4D).

We previously observed that BCL2 overexpression protects cells from the BH3-mimetic ABT-737 and that BCL2 serine 70 phosphorylation augments this protection. ${ }^{26}$
Others have demonstrated that the BCL2 mutant S70A and S70E proteins more effectively protect cells from chemotherapeutic agents compared to WT BCL2. ${ }^{27}$ In our study, we tested the ability of exogenous overexpression of WT BCL2, BCL2S70A, and BCL2S70E to protect MOLM13 cells from ONO-7475. Total viable EV control MOLM13 cells treated with $30 \mathrm{nM} \mathrm{ABT-199}$ were reduced by $35 \%$ compared to vehicle-treated cells (Figure 5A). MOLM13 cells overexpressing exogenous WT or mutant BCL2 were slightly more resistant, demonstrating viable cell reductions of $\sim 22 \%, 14 \%$, and $8 \%$ for WT, S70A, and S70E, respectively. Additionally, WT BCL2 OE slightly protected cells from $10 \mathrm{nM}$ ONO-7475, while mutant BCL2 OE rendered MOLM13 cells more resistant to this inhibitor (Figure 5A). Exogenous WT and mutant BCL2 similarly protected cells from apoptosis (Figure $5 B$ ). We next examined the effect of BCL2 overexpression on AXL expression. In MOLM13 cells, increased BCL2 expression resulted in $A X L$ induction (Figure $5 C$ and D). In fact, WT BCL2 OE or mutant BCL2 OE (both S7OA and S70E) each mediated a 
A

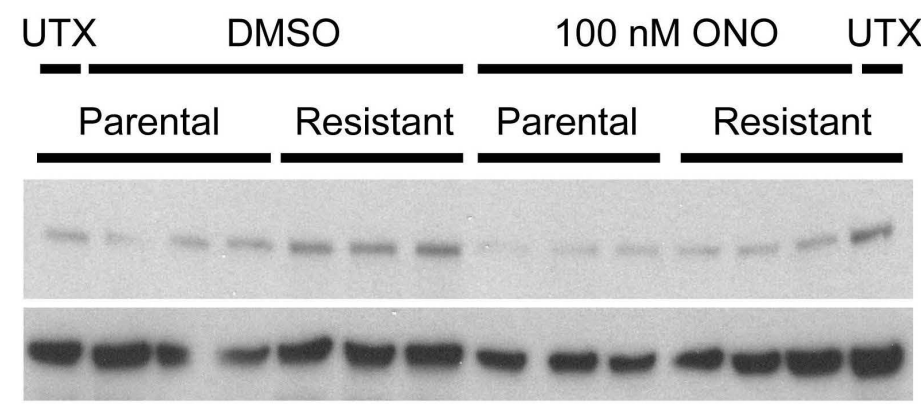

BRAF

Tubulin
BRAF

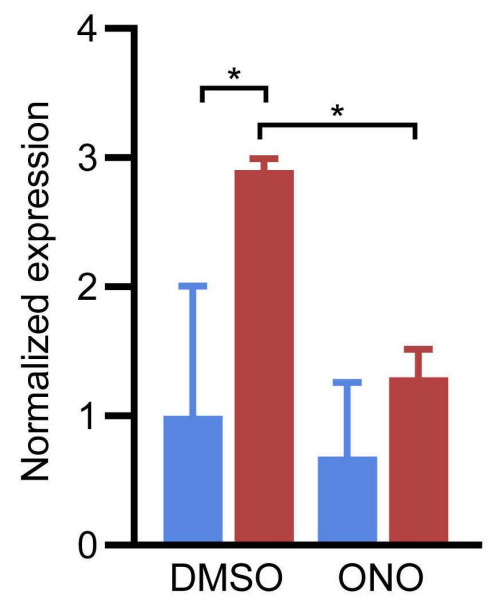

MV4;11 Parental

MV4;11 ABT-199

Resistant
B
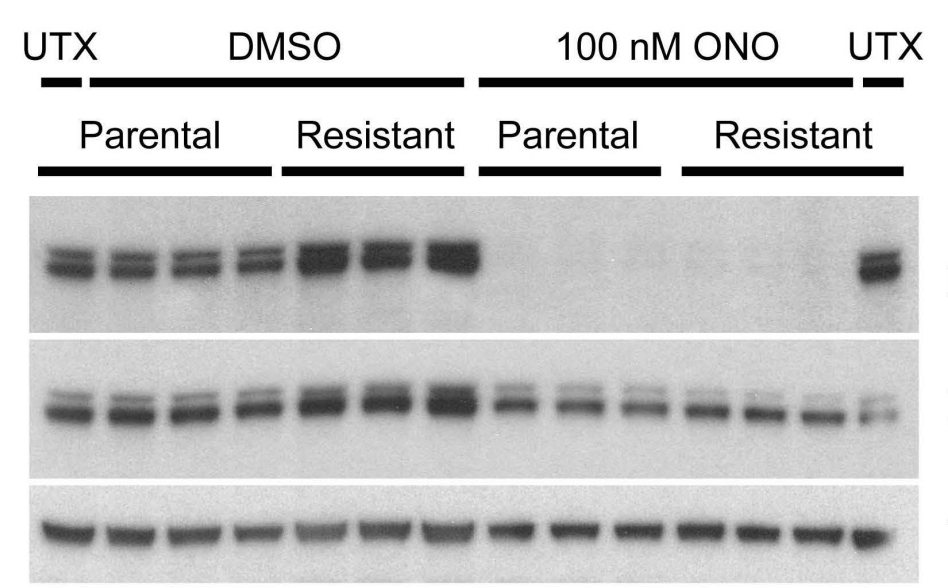

p-ERK

Tubulin

C
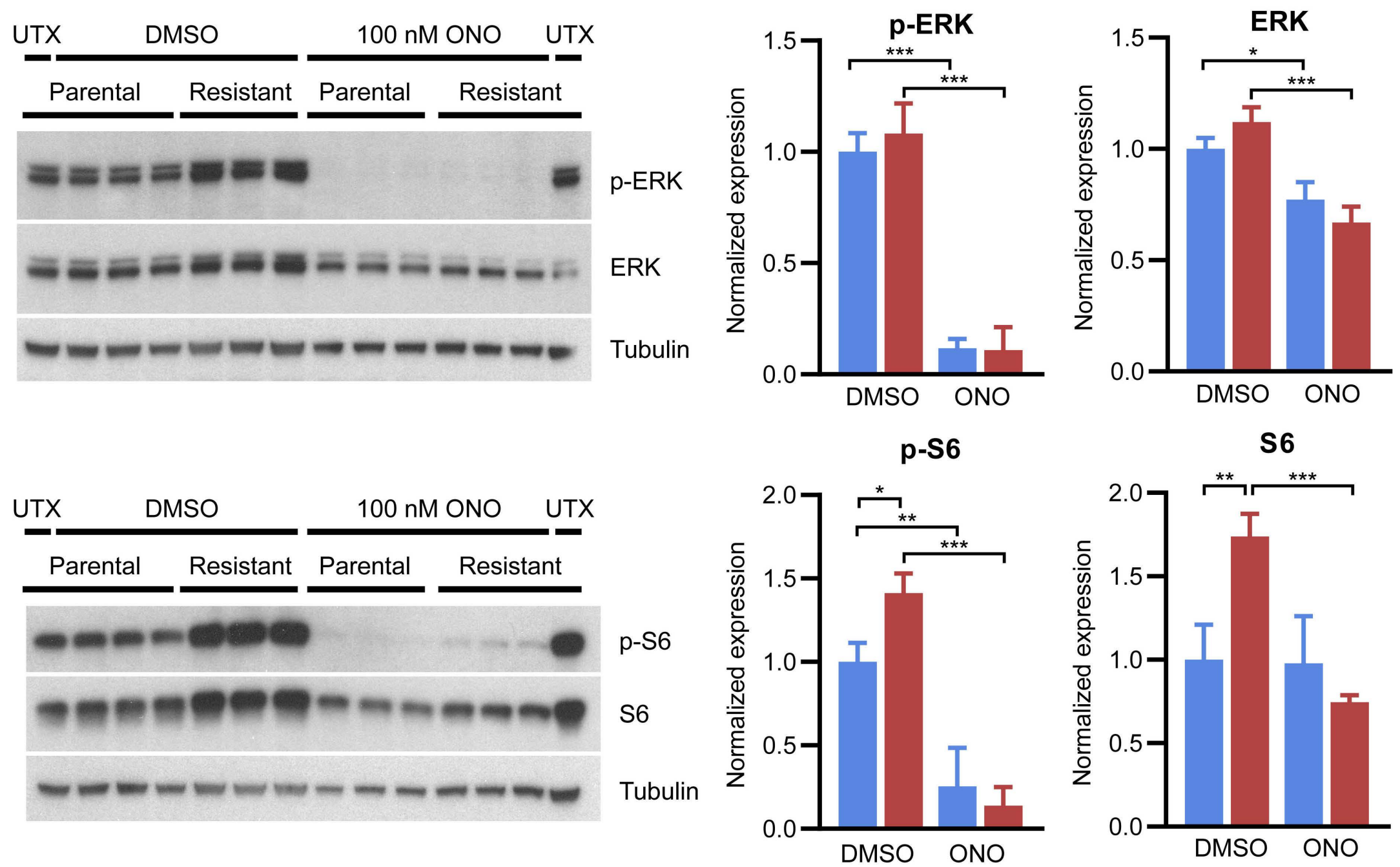

D

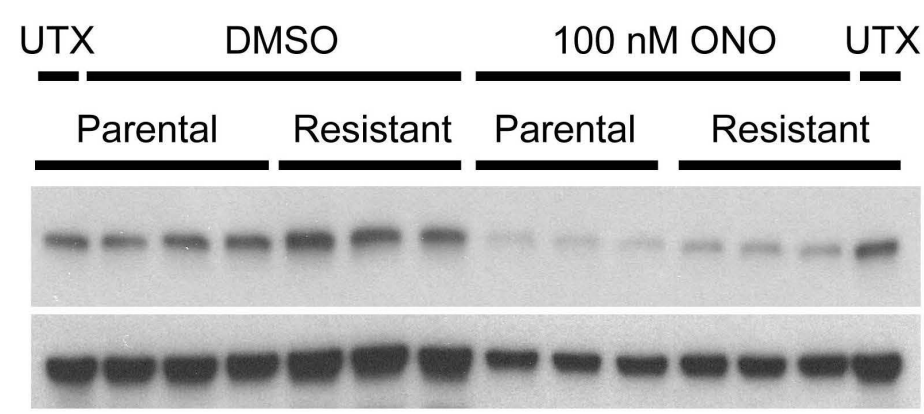

p-S6

S6

Tubulin

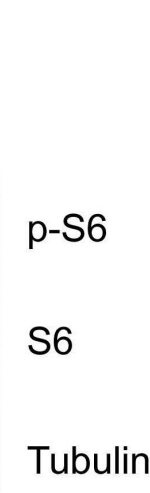

MCL-1

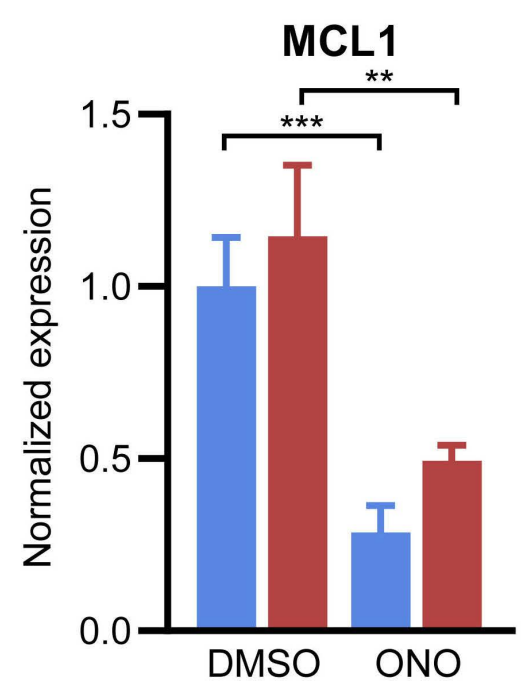

MV4;11 Parental MV4;11 ABT-199

Resistant

Figure 3. ABT ONO-7475 suppresses pro-growth and pro-survival targets in parental and ABT-199-resistant cells. MV4;11 parental and ABT-199-resistant cells were treated with $100 \mathrm{nM}$ ONO-7475 for 24 hours. Expression changes in B-RAF (A), ERK and p-ERK (B), S6 and p-S6 (C), and MCL-1 (D) in MV4;11 parental and resistant cells were determined via western blotting. Tubulin serves as a control. ABT-199-mediated and ONO-7475 dependent expression changes are represented in accompanying bar graphs. Densitometry was performed using ImageJ. One-way ANOVA with Dunnett's post-test was performed to determine significance $(* P<0.033 ; * * P<0.002 ; * \star \star P<0.001)$. 
A

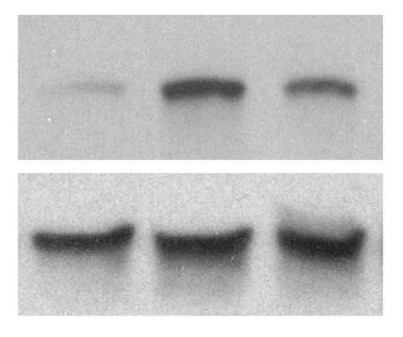

MCL-1

$\beta$-tubulin

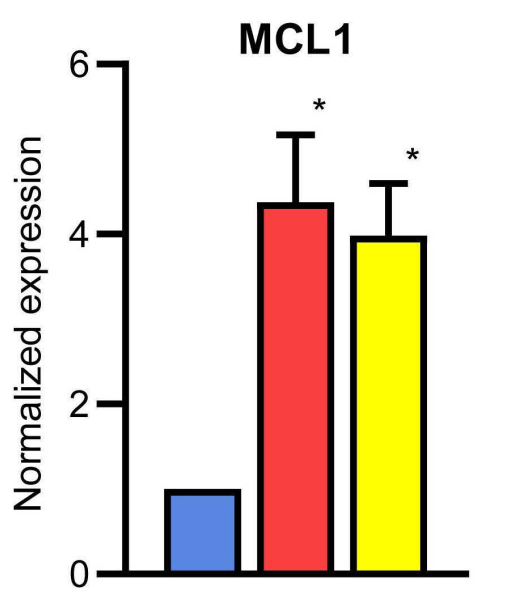

B

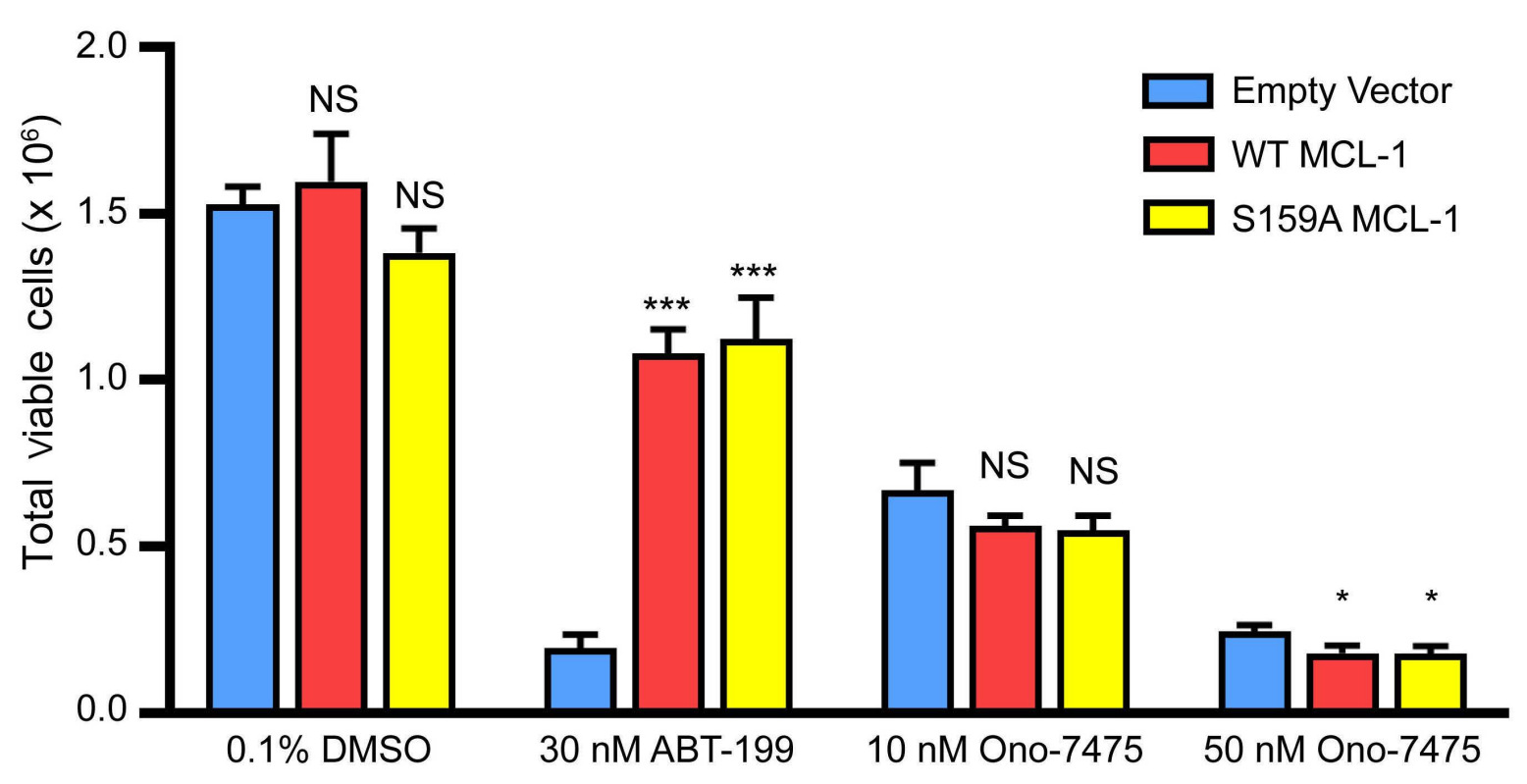

C

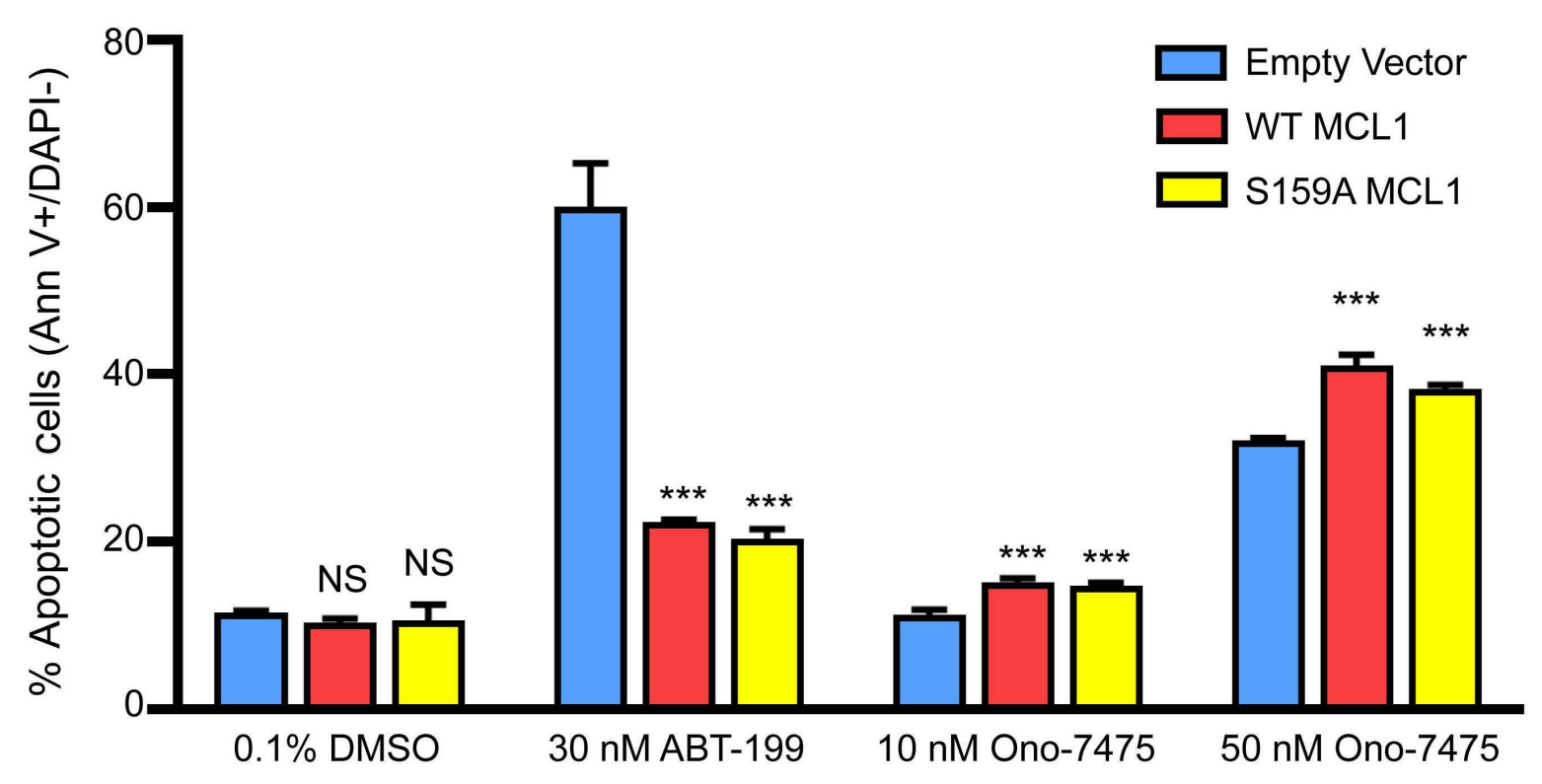

D

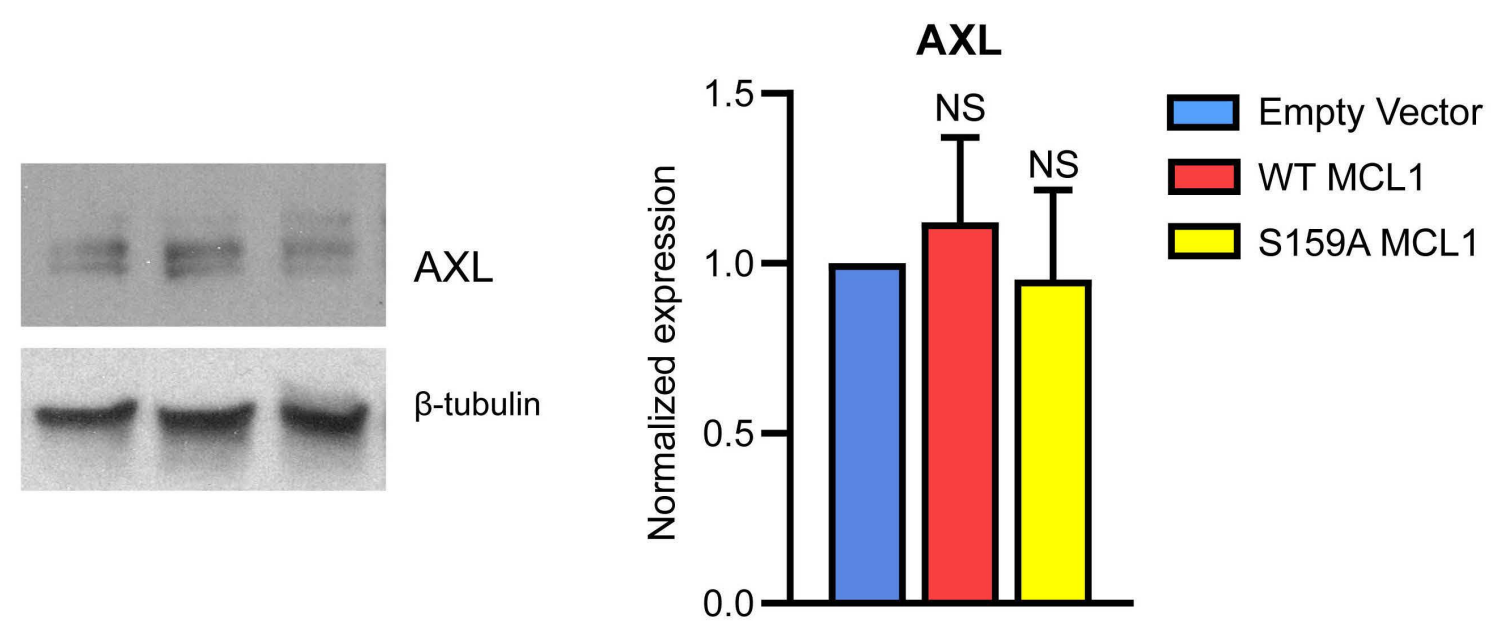

Figure 4. MV4;11 cells overexpressing WT or gain of function S159A mutant MCL-1 are resistant to $A B T-199$ but are similarly sensitive to ONO7475 compared to control cells. MV4;11 control (Empty Vector; EV) and MV4;11 cells overexpressing WT MCL-1 or S159A mutant MCL-1 were treated with vehicle $(0.1 \%$ DMSO), $10 \mathrm{nM}$, or $50 \mathrm{nM}$ ONO7475 for 72 hours. One-way ANOVA with Dunnett's posttest was performed to determine significance $(* P<0.033$; $\star \star P<0.002 ; * \star * P<0.001) \ln (\mathrm{A})$ western blot of MCL-1 expression levels in EV control, WT MCL-1 OE, and S159A mutant MCL-1 OE are depicted. Viable total cells $(B)$ and percentage of apoptotic cells (C) were determined by flow cytometry using counting beads, Annexin $\mathrm{V}$, and DAPI. (D) AXL expression in MV4;11 cells based on MCL-1 expression levels. Densitometry was performed by normalizing to tubulin using ImageJ. 
significant increase in $A X L$ expression that was alleviated by treatment with ABT-199 (Figure 5D).

\section{The combination of ONO-7475 and ABT-199 is effective in FLT3-ITD AML MOLM13 cell line xenograft and the FLT3-ITD AML PDX model}

To examine in vivo efficacy of this combination, we injected $6 \times 10^{5}$ MOLM13 luc/gfp cells into NSG mice $(n=35)$. Once leukemia burden was established by IVIS imaging, the mice were split into four groups: control $(0.1 \%$ Tween80; $n=10$ ), ONO-7475 (10 mg/kg; $n=8) ; A B T-199$ (100 $\mathrm{mg} / \mathrm{kg} ; \mathrm{n}=8)$; and combination $(\mathrm{n}=9)$. The average survival of mice in the control group was 16 days; ABT-199 only extended survival by two days although this difference was statistically significant $(P=0.001)$. ONO-7475 was effective alone and extended survival to 22 days $(P<0.0001)$, however, the combination therapy was most efficacious (average survival 35 days, $P<0.0001$, Figure $6 \mathrm{~A}$ ). Throughout the treatment course, leukemia burden was monitored in five mice from each group via IVIS imaging (Online Supplementary Figure S6). As shown in Figure 6B, the combination of ONO-7475 and ABT-199 was effective in reducing the leukemia burden. After nine days, mean fold change in radiance increased 83 -fold in vehicle-treated mice, 45fold with ONO-7475 alone, 102-fold with ABT-199 alone, and only 10 -fold with the combination. After 16 days, the vehicle mice had all died. However, at this time, IVIS imaging revealed that the ONO-7475/ABT-199 combination group displayed a 27 -fold increase in radiance (leukemic burden) while the ONO-7475 and ABT-199 cohorts had 258-fold and 610-fold increases, respectively (Figure 6B). We next tested the efficacy of the ONO-7475/ABT-199 combination in a FLT3-ITD AML PDX model. AML PDX cells (1 million) were injected into 20 NSG mice. After verification of leukemia burden by analytical flow cytometry for human CD45 (on average 10\% human $C D 45^{+}$cells), the mice were divided into four cohorts ( $n=5$ each): vehicle (0.1\% Tween 80), $10 \mathrm{mg} / \mathrm{kg}$ ONO- 7475, $100 \mathrm{mg}$ ABT-199, and combined therapy. ONO-7475 showed efficacy as a single agent, as the average survival was 60 days in the vehicletreated cohort compared to 86 days in the ONO-7475treated cohort ( $P=0.0026$; Figure $6 \mathrm{C})$. In the PDX model, ABT-199 did not significantly extend overall survival $(P=0.0751)$. However, the combination of ONO-7475 with ABT-199 was especially effective as it extended survival by 39 days $(P=0.0026)$. Throughout the course of treatment, the leukemia burden was assessed by measuring human $C D 45^{+}$cells in the blood of mice at various intervals. As shown in Figure 6D, after six weeks, human AML cells in vehicle-treated mice were $\sim 91 \%$, while mice receiving the ONO-7475/ABT-199 combination had a leukemia burden in the blood of $\sim 27 \%$. After eight weeks, vehicle-treated mice had $\sim 98 \%$ human CD45+ cells while mice receiving the ONO-7475/ABT-199 combination had only 44\% human CD45 ${ }^{+}$cells.

We next examined the extent of tumor burden in tissues from the murine PDX transplantation model. Hematoxylin and Eosin (H\&E) staining revealed significant splenic infiltration by leukemic cells characterized morphologically by moderate amounts of cytoplasm, irregular nuclear contours, open (blastic) chromatin patterns, and numerous mitotic figures (red arrowheads in Online Supplementary Figure S7A). In both the ONO-7475 and ABT-199 treated groups, mitotic figures, as well as increased numbers of apoptotic figures (relative to control), were observed. In addition, islands of native hematopoiesis were observed in the splenic parenchyma. However, in the ONO7475/ABT-199 combination group, there was a notable reduction of leukemic cells in the mouse spleen, which showed substantial predominance of native hematopoiesis, including megakaryocytes and admixed granulocytic and erythroid precursors. To confirm the origin of the malignant cells, immunohistochemistry was performed using a human-specific anti-Ku-80 antibody that specifically recognizes the human Ku-80 protein (Online Supplementary Figure S7B). Ku-80 staining demonstrated a higher proportion of human leukemic cells in the control group compared to the ONO-7475 treated group.

\section{Discussion}

We recently demonstrated that ONO-7475 was effective as a single agent against FLT3-ITD AML cells. ${ }^{19}$ The mechanism of ONO-7475 action involved diverse targets including the inhibition of survival kinases such as ERK1/2, MCL-1, and many cell cycle regulators. Considering that MCL-1 and ERK1/2 are major factors in ABT-199 resistance, ${ }^{7-10}$ the ability of ONO-7475 to overcome ABT-199 resistance in FLT3-ITD AML cells is an uncharacterized and intriguing area of research.

ONO-7475 as a single agent was effective against both MV4;11 and MOLM13 cells. However, its combination with ABT-199 demonstrated significant synergy and resulted in near elimination of the leukemic cells in both cell lines (Figure $1 \mathrm{~A}$ and B and Online Supplementary Figure S1). These same synergistic effects were also observed in primary patient samples (Figure 1D). While there were some differences in apoptosis between patient samples when single agents were used (ONO-7475, more effective in Patient \#28 or ABT199 more effective in Patient \#32), both samples were extremely sensitive to the combination of the two agents.

Additionally, the notion that ONO-7475 may be useful in FLT3-ITD (or TKI-resistant) AML is highlighted by the cellular sensitivity of patient \#28 compared to patient \#32; this patient harbored a FLT3 D835 mutation which render cells resistant to the majority of TKIs in the clinic. ${ }^{1}$ While treatment of mutant FLT3 is challenging in AML, new 
A

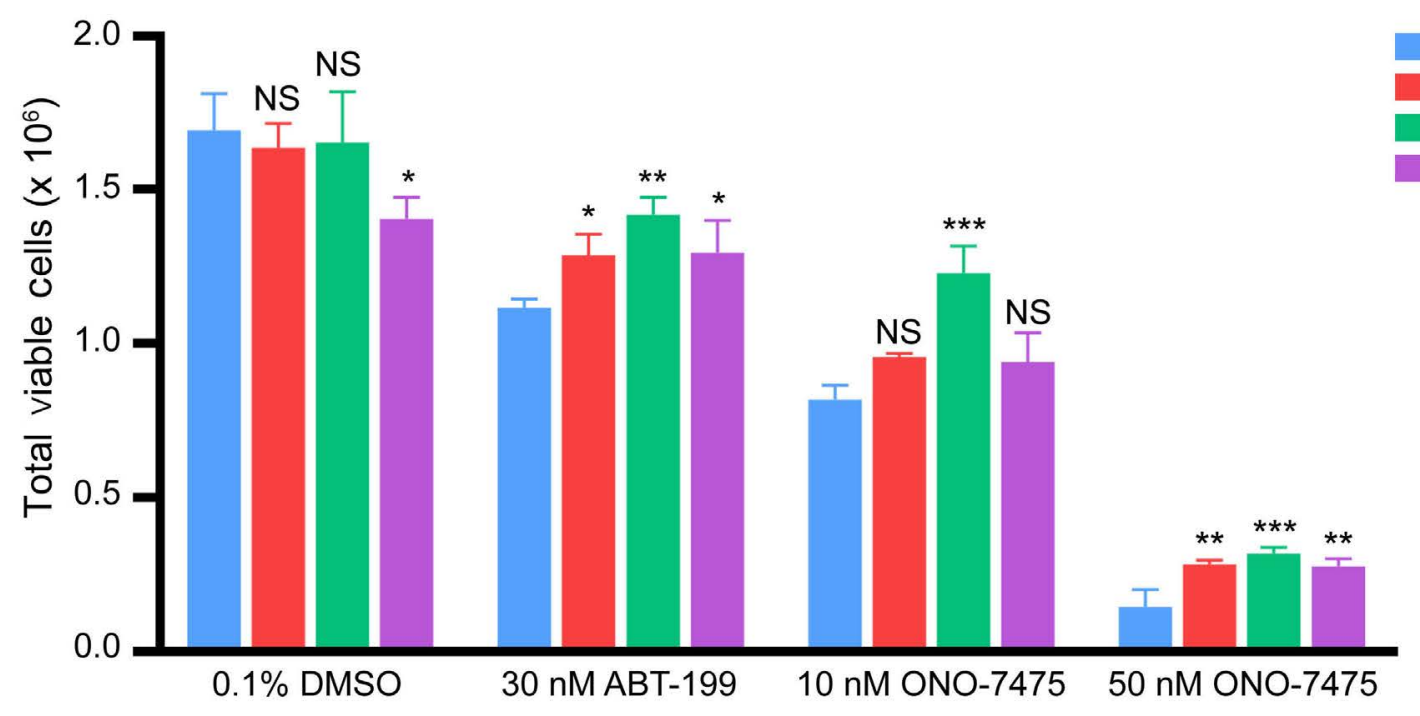

B

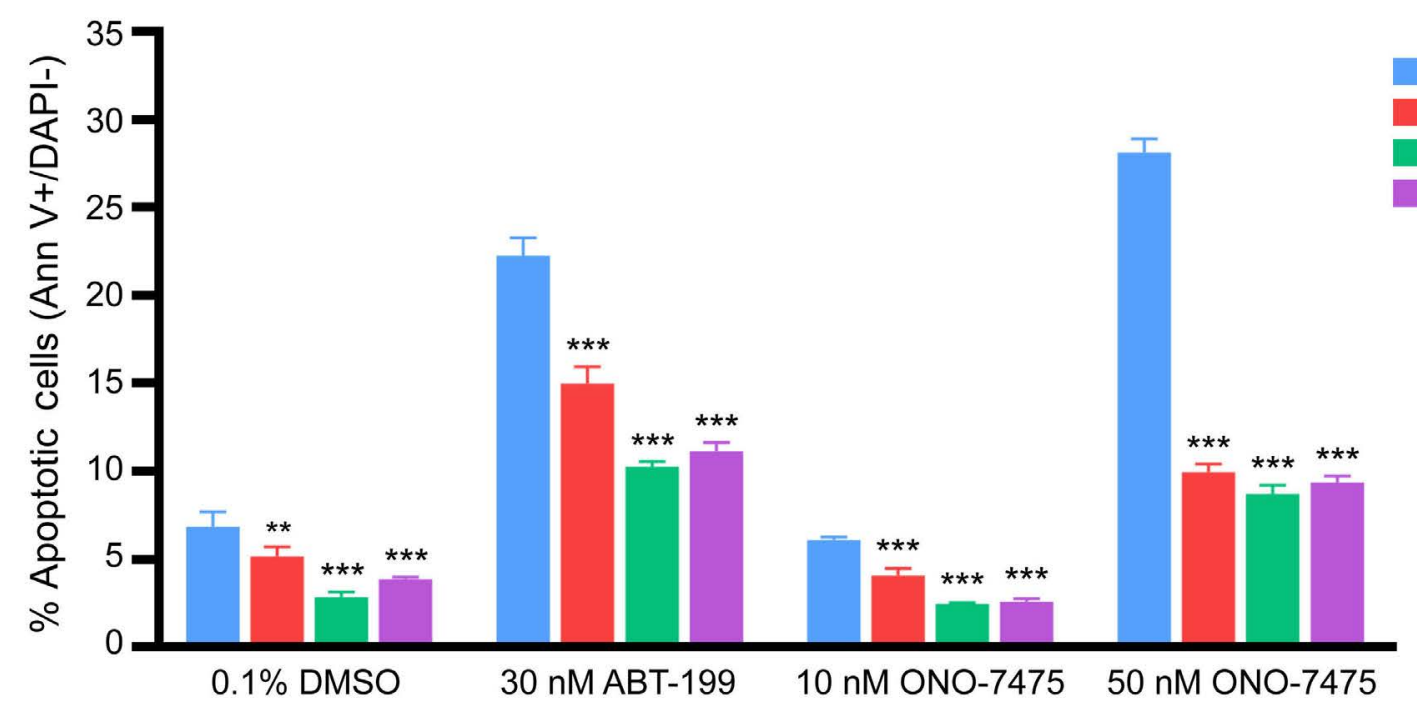

Molm13 norm to ABL1

$\mathrm{BCL} 2$

C

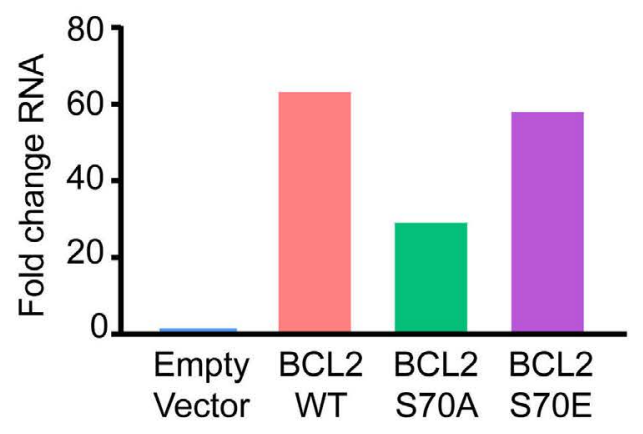

Molm13 norm to ABL1

D

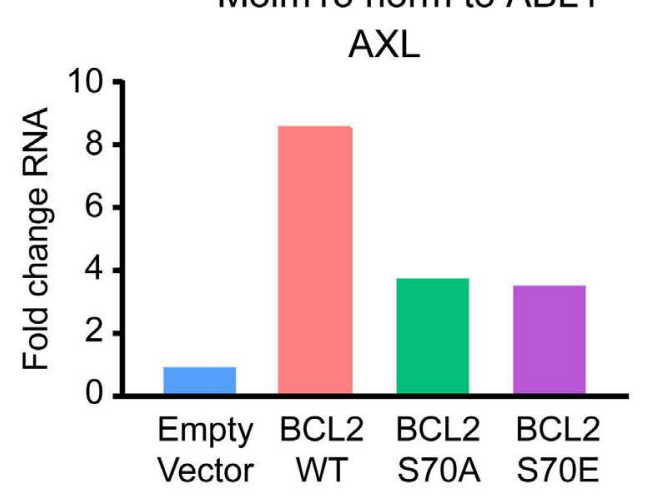

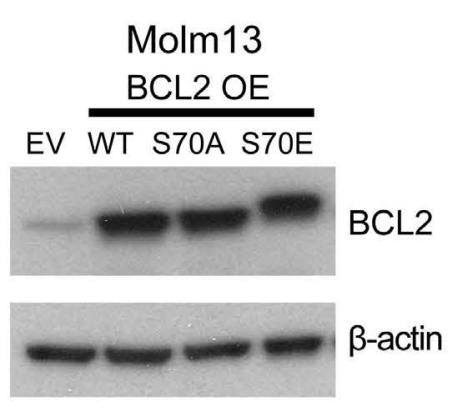

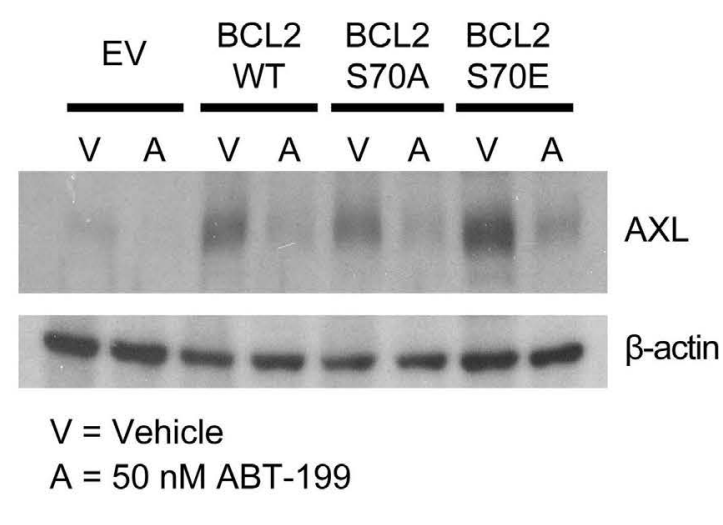

Empty Vector

WT BCL2

S70A BCL2

S70E BCL2
Empty Vector

WT BCL2

S70A BCL2

S70E BCL2

BCL2

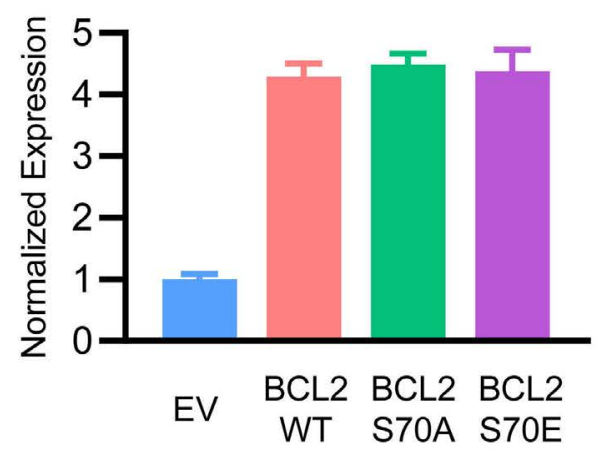

AXL

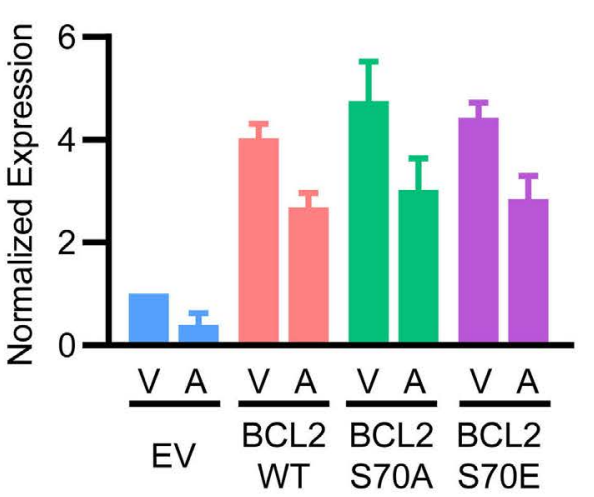

Figure 5. Overexpression of WT or gain of function S70A or S70E BCL2 mutants induces AXL expression and protects MOLM13 cells from ONO-7475. MOLM13 control (Empty Vector; EV) and MOLM13 cells overexpressing WT BCL2 or S70A mutant or S70E mutant BCL2 were treated with vehicle (0.1\% DMSO), $10 \mathrm{nM}$, or $50 \mathrm{nM}$ ONO-7475 for 72 hours. Viable total cells (A) and percentage of apoptotic cells (B) were determined by flow cytometry using counting beads, Annexin V, and DAPI. One-way ANOVA with Dunnett's post-test was performed to determine significance $(* P<0.033 ; * \star P<0.002 ; * \star * P<0.001)$. (C) Expression of BCL2 WT, S70A mutant, or S70E mutant was determined in MOLM13 cells. RNA and protein were isolated and BCL2 expression was monitored by qRT-PCR and western blot, respectively. (D) Expression of AXL was determined in MOLM13 cells expressing WT, S70A mutant, or S70E mutant BCL2. RNA and protein were isolated and BCL2 and AXL expression was monitored by qRT-PCR and western blot, respectively. Densitometry was performed using ImageJ. 
agents that target the FLT3/AXL axis have shown efficacy. As such, Gilteritinib, a dual FLT3/AXL inhibitor, has shown activity in FLT3-mutant AM but it has been shown to activate ERK signaling and promote RAS mutations. Thus, novel agents, such as ONO-7475s that specifically target AXL signaling, may be useful to augment (or used in a resistant setting) as it does not result in ERK activation.

Previously, we demonstrated that low-dose ONO-7475 directly impacts the expression of pro-growth proteins. ${ }^{19}$ Here, we demonstrate that $\mathrm{p}$-ERK levels were reduced in an ONO-7475-dependent manner (Figure 3B). This is a critical observation as ERK signaling supports MCL-1 stability via phosphorylation of threonine 92 and 163, resulting in MCL-1 stabilization. ${ }^{28,29}$ Thus, the mechanisms by which ERK signaling and AXL inhibitors impact the regulation of MCL-1 warrants further investigation.

To better understand how ABT-199 resistance impacts cellular programs, we analyzed differences between the proteomic profile of parental and ABT-199 resistance MV4;11 cells. Several cell cycle and pro-survival proteins were altered in the untreated parental and resistant cell lines. While many of these alterations portend for poor outcomes, they may also offer potential targets in ABT-199-resistant AML (Figure 3A-D and Online Supplementary Figures S4 and S5). A comparison of proteomic profiles of MV4;11 parental and MV4;11 ABT-199-resistant cells treated with 100 nM ONO-7475 for 24 hours revealed that the agent had similar effects on targets in both cell lines. Here, we ob-
A

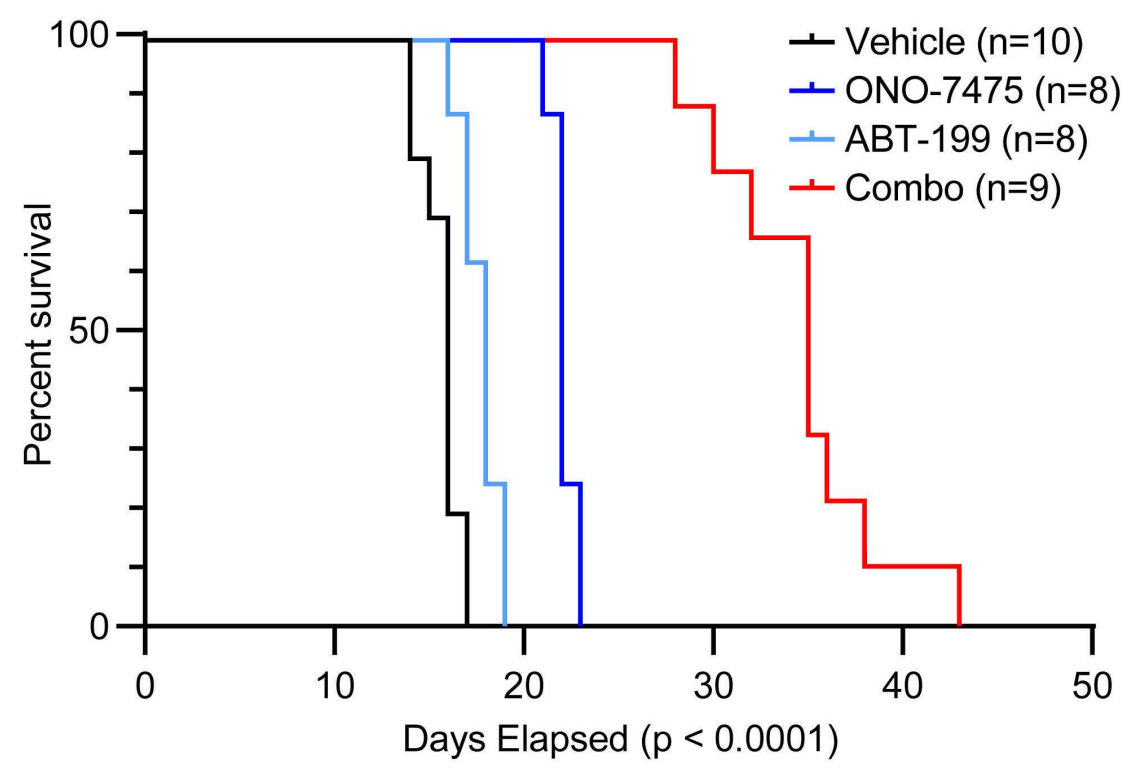

C

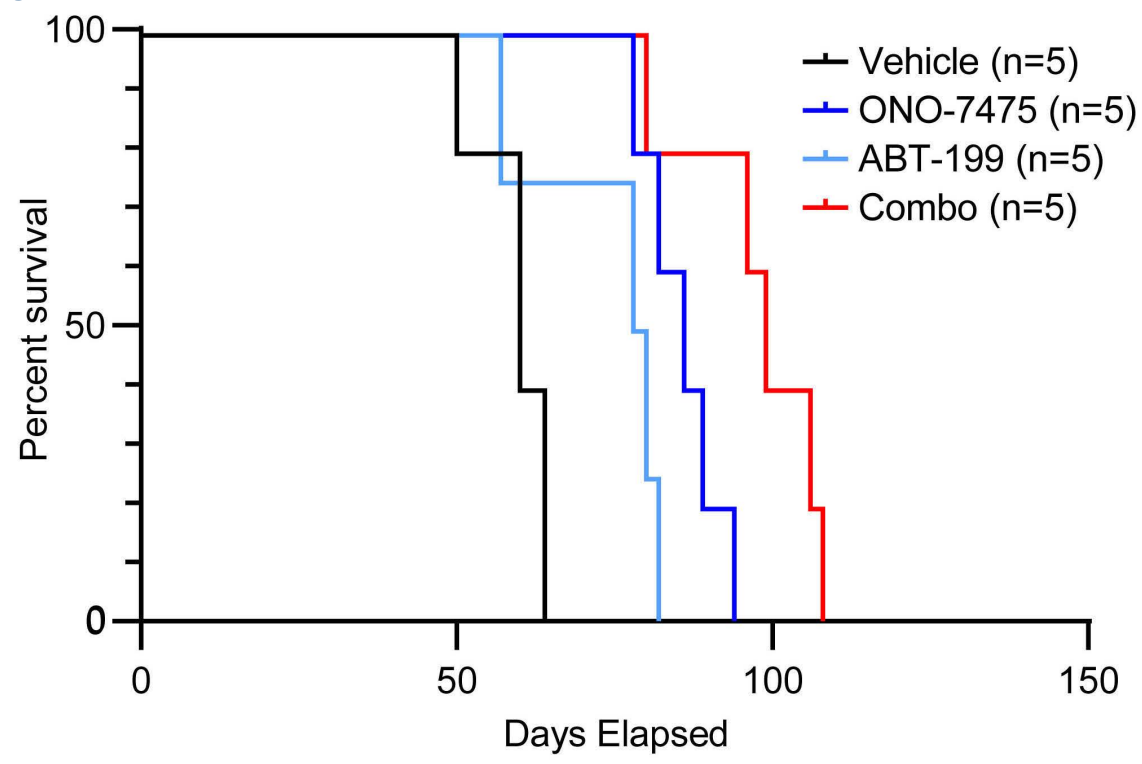

B

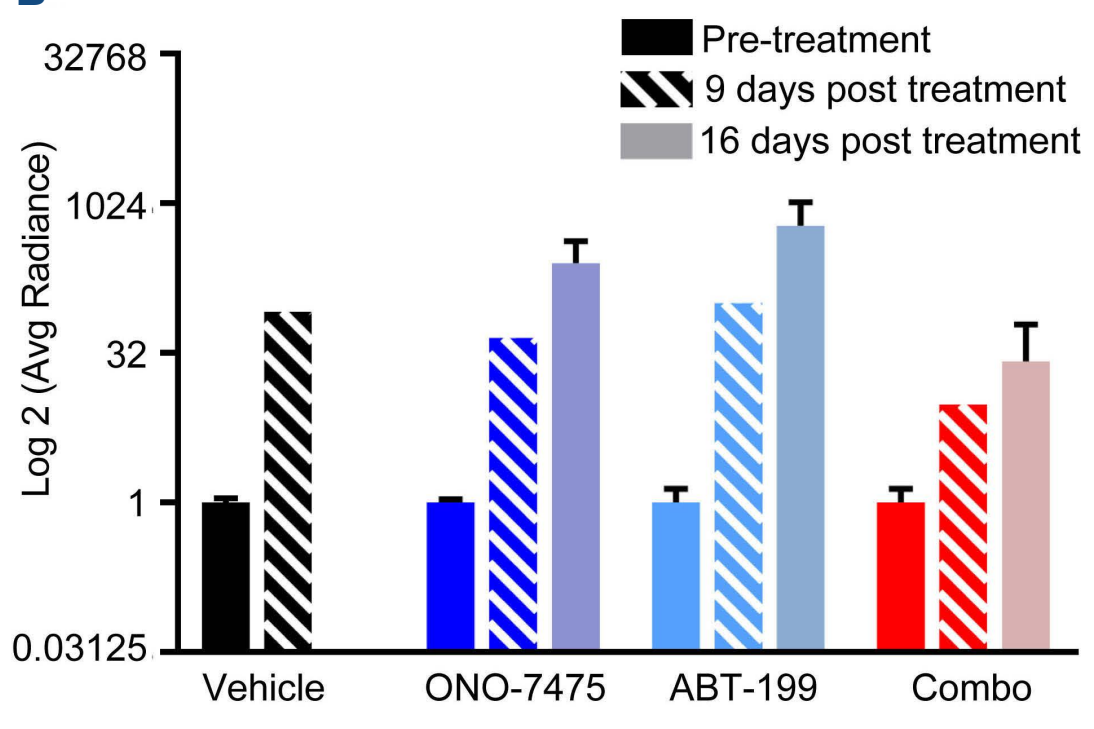

D

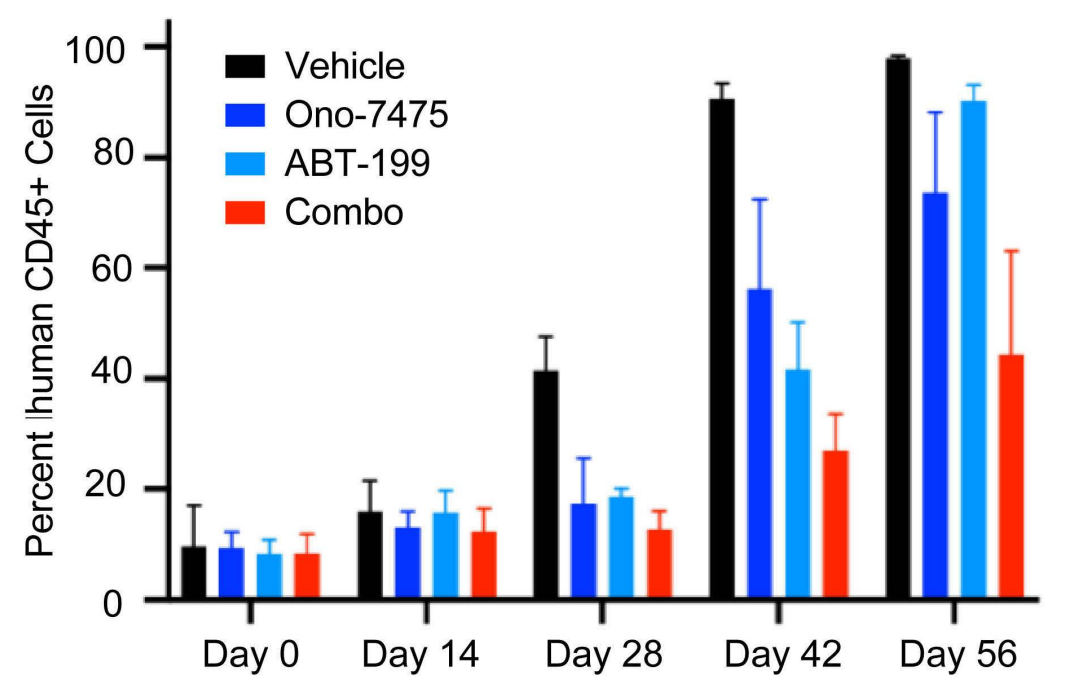

Figure 6. ONO-7475 combination is effective in reducing leukemia burden and promoting mouse survival in xenograft models using a FLT3-ITD AML cell line and a FLT3-ITD AML PDX. MOLM13 luc/gfp cells were injected into NSG mice. After leukemia engraftment, determined by IVIS imaging, mice were treated with vehicle (Tween 80 ), $10 \mathrm{mg} / \mathrm{kg}$ ONO-7475, $100 \mathrm{mg} / \mathrm{kg}$ ABT-199 or a combination of both agents. (A) Survival data with median survival (days) is indicated for each group. (B) Measurement of fold change radiance during the course of treatment using IVIS imaging software. (C) Survival data with median survival (days) is indicated for each group. AML PDX model 3028566 cells were injected into NSG mice. After leukemia engraftment determined by flow cytometry for human CD45-positive cells, mice were treated with vehicle (Tween 80), 10 mg/kg ONO-7475, 100 mg/kg ABT199 or a combination of both agents. (D) Measurement of leukemia burden by measuring CD45-positive cells by flow cytometry during the course of treatment. 
served that ONO-7475 similarly suppressed cell cycle regulators (e.g., p-ERK, S6, p-S6, and B-RAF) and pro-survival proteins (e.g., MCL1) in parental and ABT-199-resistant cells (Figure 3 and Online Supplementary Figures S4 and S5). These results suggest that ONO-7475 remains effective in inhibiting potential ABT-199-resistant pathways.

MCL-1 upregulation is a known resistance mechanism for ABT-199. Consistently, we observed that MV4;11 cells overexpressing WT or GOF mutant MCL-1 were resistant to ABT-199 compared to EV-control cells. However, ONO7475 was equally as effective reducing viable cell numbers and inducing apoptosis in EV-control cells and cells that overexpress either WT or GOF mutant MCL-1 (Figure 4B and $4 C$ ). Interestingly, overexpression of WT $\mathrm{BCL} 2$ or GOF mutants did impart some protection against ONO-7475 (Figure 5A and 5B), as cells overexpressing $B C L 2$ have elevated $A X L$ levels compared to EV-control cells (Figure $5 C$ and $5 D$ ). Our observation that BCL2 overexpression causes increased levels of $A X L$ (Figure $5 C$ and $5 D)$ seems to indicate that $B C L 2$ may regulate $A X L$ levels at the transcriptional level. While the mechanism for this upregulation is poorly understood, it is plausible that AXL expression is potentially regulated through the BCL2/RAS/RAF axis. This notion is supported by the findings that RAF is a target for BCL2 binding. ${ }^{30,31}$ and that RAS regulates $A X L$ in drug resistance studies in solid tumors. ${ }^{32-34}$ These observations warrant further investigation.

To investigate the in vivo efficacy of the ONO-7475/ABT199 combination, we utilized both cell line xenograft and FLT3-ITD AML PDX models. While ONO-7475 alone was efficacious in every setting, the combination was significantly more potent in reducing leukemia burden and prolonging the survival of mice compared to either single agent in both models. In the MOLM13 model, IVIS imaging revealed that mice treated with both agents had a much lower leukemia burden compared to vehicle-treated mice or mice treated with either single agent (Figure 6B and Online Supplementary Figure S6). Importantly, the combination of ONO-7475 and ABT-199 significantly prolonged survival (Figure 6A). Similar results were observed in an AML FLT3-ITD PDX model, where mice given both ONO7475 and ABT-199 survived longer compared to vehicletreated or either single agent and displayed reduced leukemia burden as observed by human $C D 45^{+}$cells (Figure $6 \mathrm{C}$ and $6 \mathrm{D}$ ). It is worth noting that the PDX model could, possibly, represent a high-risk AML model in view of the concomitant presence of FLT-ITD, NPM1, and DNMT3A mutations, as reported previously. ${ }^{35}$

Several clinical studies have demonstrated a favorable role for ABT-199 in AML therapy, but primary resistance and subsequent treatment failure remain common. In a recent study, primary or subsequent resistance to venetoclax-based treatment regimens appeared to associate with the presence of activating mutations involving FLT3 or the RAS-ERK pathway. ${ }^{36}$ This result, coupled with our findings of activation of the RAS pathway, suggests a potential resistance mechanism that may be overcome by ONO-7475. Further, our results suggest AXL/MERTK inhibition effectively synergizes with ABT-199 in AML and bypasses various mechanisms of ABT-199 resistance.

In summary, our results show that inhibition of the AXL/MERTK axis exerts a synergistic effect alongside BCL2 inhibition in AML and can overcome the survival advantages conferred by MCL-1 overexpression, a common mechanism of ABT-199 resistance. These results provide pre-clinical support for exploring this strategy in AML patients.

\section{Disclosures}

Ono Pharmaceutical supported the work performed in this study. Tomoko Yasuhiro and Ryohei Kozaki are employees of Ono Pharmaceutical.

\section{Contributions}

$P R$ and $S P$ designed experiments, performed research, analyzed data, and wrote the manuscript; PM, HM, MA, $P Y M, X Z, V R$, and $B C$ performed research, and analyzed data; $M K, C D, M A$ and JK analyzed data and helped edit the manuscript; TY and RK provided compound, helped with experimental design, and helped edit the manuscript.

\section{Acknowledgments}

The authors would like to express their profound respect and admiration for Dr Peter P. Ruvolo who passed away as this manuscript was going through peer review. The work in this study would not have taken place were it not for Peter's tireless work and dedication to science and discovery. He is survived by his wife, sister, and numerous family members.

\section{Funding}

This work was supported by Ono Pharmaceutical Co. Ltd., Research Center of Oncology, Osaka, Japan. Flow Cytometry (MA) and RPPA Cores are funded by $\mathrm{NCl}$ Cancer Center Support Grant P30CA16672. S.M.P. is supported by funding from the National Cancer Institute/National Institutes of Health Award (R01CA207204) and Leukemia and Lymphoma Society (6577-19); P.M. is supported by grants from the Jane Coffin Childs Medical Trust Fund and the American Society of Hematology. We would like to thank Rithica Deepak and Oscar Benitez for carefully reviewing this manuscript. 


\section{References}

1. Short NJ, Kantarjian H, Ravandi F, Daver N. Emerging treatment paradigms with FLT3 inhibitors in acute myeloid leukemia. Ther Adv Hematol. 2019;10:2040620719827310.

2. Roskoski R Jr. The role of small molecule Flt3 receptor proteintyrosine kinase inhibitors in the treatment of Flt3-positive acute myelogenous leukemias. Pharmacol Res. 2020;155:104725.

3. Kiyoi H, Kawashima N, Ishikawa Y. FLT3 mutations in acute myeloid leukemia: Therapeutic paradigm beyond inhibitor development. Cancer Sci. 2020;111(2):312-322.

4. Smith CC. The growing landscape of FLT3 inhibition in AML. Hematology Am Soc Hematol Educ Program. 2019;2019(1):539547.

5. Lachowiez C, DiNardo CD, Konopleva M. Venetoclax in acute myeloid leukemia - current and future directions. Leuk Lymphoma. 2020;61(6):1313-1322.

6. Konopleva M, Pollyea DA, Potluri J, et al. Efficacy and biological correlates of response in a Phase II study of venetoclax monotherapy in patients with acute myelogenous leukemia. Cancer Discov. 2016;6(10):1106-1117.

7. Pan R, Hogdal LJ, Benito JM, et al. Selective BCL-2 inhibition by ABT-199 causes on-target cell death in acute myeloid leukemia. Cancer Discov. 2014;4(3):362-375.

8. Pan R, Ruvolo VR, Wei J, et al. Inhibition of Mcl-1 with the panBcl-2 family inhibitor (-)BI97D6 overcomes ABT-737 resistance in acute myeloid leukemia. Blood. 2015;126(3):363-372.

9. Lin KH, Winter PS, Xie A, et al. Targeting MCL-1/BCL-XL forestalls the acquisition of resistance to ABT-199 in acute myeloid leukemia. Sci Rep. 2016;6:27696.

10. Pan R, Ruvolo V, Mu H, et al. Synthetic lethality of combined Bcl-2 inhibition and p53 activation in AML: mechanisms and superior antileukemic efficacy. Cancer Cell. 2017;32(6):748-760.

11. Ramsey HE, Fischer MA, Lee T, et al. A novel MCL1 inhibitor combined with venetoclax rescues venetoclax-resistant acute myelogenous leukemia. Cancer Discov. 2018;8(12):1566-1581.

12. Wei AH, Roberts AW, Spencer A, et al. Targeting MCL-1 in hematologic malignancies: Rationale and progress. Blood Rev. 2020;44:100672.

13. Yoshimoto G, Miyamoto T, Jabbarzadeh-Tabrizi S, et al. FLT3-ITD up-regulates MCL-1 to promote survival of stem cells in acute myeloid leukemia via FLT3-ITD-specific STAT5 activation. Blood. 2009;114(24):5034-5043.

14. Ma J, Zhao S, Qiao X, et al. Inhibition of Bcl-2 synergistically enhances the antileukemic activity of Midostaurin and Gilteritinib in preclinical models of FLT3-mutated acute myeloid leukemia. Clin Cancer Res. 2019;25(22):6815-6826.

15. McMahon CM, Ferng T, Canaani J, et al. Clonal selection with RAS pathway activation mediates secondary clinical resistance to selective FLT3 inhibition in acute myeloid leukemia. Cancer Discov. 2019;9(8):1050-1063.

16. Park IK, Mishra A, Chandler J, et al. Inhibition of the receptor tyrosine kinase Axl impedes activation of the FLT3 internal tandem duplication in human acute myeloid leukemia: implications for Axl as a potential therapeutic target. Blood. 2013;121(11):2064-2073.

17. Ben-Batalla I, Schultze A, Wroblewski M, et al. Axl, a prognostic and therapeutic target in acute myeloid leukemia mediates paracrine crosstalk of leukemia cells with bone marrow stroma. Blood. 2013;122(14):2443-2452.

18. Park IK, Mundy-Bosse B, Whitman SP, et al. Receptor tyrosine kinase $A x l$ is required for resistance of leukemic cells to FLT3targeted therapy in acute myeloid leukemia. Leukemia.
2015;29(12):2382-2389.

19. Ruvolo PP, Ma H, Ruvolo VR, et al. Anexelekto/MER tyrosine kinase inhibitor ONO-7475 arrests growth and kills FMS-like tyrosine kinase 3-internal tandem duplication mutant acute myeloid leukemia cells by diverse mechanisms. Haematologica. 2017;102(12):2048-2057.

20. Gallardo M, Lee HJ, Zhang X, et al. hnRNP K Is a haploinsufficient tumor suppressor that regulates proliferation and differentiation programs in hematologic malignancies. Cancer Cell. 2015;28(4):486-499.

21. Chou TC, Talalay P. Quantitative analysis of dose-effect relationships: the combined effects of multiple drugs or enzyme inhibitors. Adv Enzyme Regul. 1984;22:27-55.

22. Dumas P-Y, Naudin C, Martin-Lannerée S, et al. Hematopoietic niche drives FLT3-ITD acute myeloid leukemia resistance to quizartinib via STAT5-and hypoxia-dependent upregulation of AXL. Haematologica. 2019;104(10):2017-2027.

23. McCubrey JA, Steelman LS, Chappell WH, et al. Ras/Raf/MEK/ERK and PI3K/PTEN/Akt/mTOR cascade inhibitors: how mutations can result in therapy resistance and how to overcome resistance. Oncotarget. 2012;3(10):1068-1111.

24. Domina AM, Vrana JA, Gregory MA, et al. MCL1 is phosphorylated in the PEST region and stabilized upon ERK activation in viable cells, and at additional sites with cytotoxic okadaic acid or taxol. Oncogene. 2004;23(31):5301-5315.

25. Ruvolo PP. GSK-3 as a novel prognostic indicator in leukemia. Adv Biol Regul. 2017;65:26-35.

26. Konopleva M, Contractor R, Tsao T, et al. Mechanisms of apoptosis sensitivity and resistance to the BH3 mimetic ABT-737 in acute myeloid leukemia. Cancer Cell. 2006;10(5):375-388.

27. Dai $\mathrm{H}$, Ding $\mathrm{H}$, Meng $X W$, et al. Contribution of $\mathrm{Bcl}-2$ phosphorylation to Bak binding and drug resistance. Cancer Res. 2013;73(23):6998-7008.

28. Ding Q, Huo L, Yang J-Y, et al. Down-regulation of myeloid cell leukemia-1 through inhibiting Erk/Pin 1 pathway by sorafenib facilitates chemosensitization in breast cancer. Cancer Res. 2008;68(15):6109-6117.

29. Thomas LW, Lam C, Edwards SW. Mcl-1; the molecular regulation of protein function. FEBS Lett. 584(14);2981-2989.

30. Deng X, Kornblau SM, Ruvolo PP, et al. Regulation of Bcl2 phosphorylation and potential significance for leukemic cell chemoresistance. J Natl Cancer Inst Monogr. 2001;(28):30-37.

31. Wang HG, Rapp UR, Reed JC. Bcl-2 targets the protein kinase Raf-1 to mitochondria. Cell. 1996;87(4):629-638.

32. Nelson-Taylor SK, Le AT, Yoo M, et al. Resistance to RETinhibition in RET-rearranged NSCLC is mediated by reactivation of RAS/MAPK signaling. Mol Cancer Ther. 2017;16(8):1623-1633.

33. Bockorny B, Rusan M, Chen W, et al. RAS-MAPK reactivation facilitates acquired resistance in FGFR1-amplified lung cancer and underlies a rationale for upfront FGFR-MEK blockade. Mol Cancer Ther. 2018;17(7):1526-1539.

34. Brand TM, lida M, Stein AP, et al. AXL mediates resistance to cetuximab therapy. Cancer Res. 2014;74(18):5152-5164.

35. Wang YW, Tsai CH, Lin CC, et al. Cytogenetics and mutations could predict outcome in relapsed and refractory acute myeloid leukemia patients receiving BCL-2 inhibitor venetoclax. Ann Hematol. 2020;99(3):501-511.

36. DiNardo CD, Tiong IS, Quaglieri A, et al. Molecular patterns of response and treatment failure after frontline venetoclax combinations in older patients with AML. Blood. 2020;135(11):791-803. 